\title{
Topics in Nevanlinna Theory
}

\author{
Matthew M. Buck, MMath.
}

Thesis submitted to The University of Nottingham

for the degree of Doctor of Philosophy

July 2013 


\begin{abstract}
Nevanlinna Theory is a powerful quantitative tool used to study the growth and behaviour of meromorphic functions on the complex plane. It plays an important role in value distribution theory, including generalising Picard's theorem that an entire function which omits two finite values is constant. The Nevanlinna Characteristic $T(r, f)$ is a measure of a function's growth, and its associated counting function estimates how often certain values are taken. Using these tools, as well as other forms of modern complex analysis, we investigate several problems relating to differential polynomials in meromorphic functions. We also present a result relating to integer-valued meromorphic functions.
\end{abstract}




\section{Contents}

1 Introduction to Nevanlinna Theory 4

1.1 Basic definitions and classical lemmas . . . . . . . . . . . . . . 4

1.2 The Nevanlinna functionals . . . . . . . . . . . . . . . . . . 7

1.3 The Fundamental Theorems . . . . . . . . . . . . . . . . . . . . . . . . 10

1.4 Differential polynomials . . . . . . . . . . . . . . . . . . 14

2 Pairs of non-homogeneous linear differential polynomials $\quad 16$

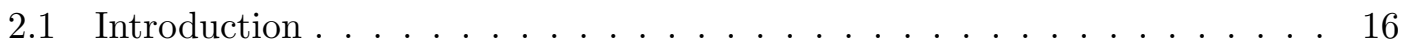

2.2 Results . . . . . . . . . . . . . . . . . . . 20

2.3 Preliminary lemmas $\ldots \ldots \ldots \ldots \ldots \ldots$

2.4 Proofs of the theorems $\ldots \ldots \ldots \ldots \ldots \ldots \ldots$

2.4 .1 Initial steps . . . . . . . . . . . . . . . . 25

2.4.2 Proof of Theorem $2.2 .1 \ldots \ldots \ldots$. . . . . . . . . . . 31

2.4 .3 Proof of Theorem $2.2 .2 \ldots \ldots \ldots$

2.4.4 Proof of Theorem $2.2 .3 \ldots \ldots \ldots$

2.4.5 Proof of Theorem $2.2 .4 \ldots \ldots \ldots$. . . . . . . . . . . 42

3 Non-linear homogeneous differential polynomials in $f$ and $f^{(k)} \quad 44$

3.1 Introduction and results . . . . . . . . . . . . . . . . . . 44 
3.2 Lemmas . . . . . . . . . . . . . . . . . . . . . . . 46

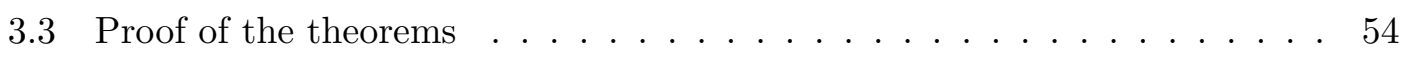

4 A result on more general homogeneous differential polynomials $\quad 56$

4.1 Introduction and result $\ldots \ldots \ldots \ldots \ldots 6$

4.2 Lemmas . . . . . . . . . . . . . . . . . . . . . . 59

4.2.1 An inequality related to a result of Zhang and Li . . . . . . . . 59

4.2 .2 Several lemmas by Whitehead . . . . . . . . . . . . . . 63

4.2 .3 Some final lemmas . . . . . . . . . . . . . . . . . . 67

4.3 Proof of Theorem $4.1 .1 \ldots \ldots \ldots \ldots$. . . . . . . . . . . . . . 69

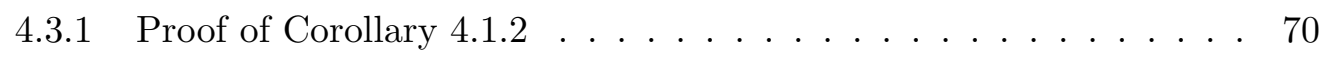

5 A normal families result for homogeneous differential polynomials $\quad 71$

5.1 Introduction and result $\ldots \ldots \ldots \ldots \ldots \ldots$

5.2 Lemmas . . . . . . . . . . . . . . . . . . 74

5.3 Proof of Theorem $5.1 .2 \ldots \ldots \ldots \ldots \ldots \ldots$

$6 \quad$ Integer points of meromorphic functions $\quad 83$

6.1 Introduction and result $\ldots \ldots \ldots \ldots \ldots$

6.2 Lemmas . . . . . . . . . . . . . . . . . . 85

6.3 Proof of Theorem $6.1 .4 \ldots \ldots \ldots \ldots \ldots$. . . . . . . . . . . 91

6.4 Appendix - How small is $\lambda(d) ? \ldots \ldots \ldots \ldots$. . . . . . . . . 94

6.5 Appendix - A thought on further work . . . . . . . . . . . 96 


\section{Chapter 1}

\section{Introduction to Nevanlinna}

\section{Theory}

\subsection{Basic definitions and classical lemmas}

We will first define some basic terms for describing functions, and from there build towards a full explanation of Nevanlinna Theory.

\section{Definitions 1.1.1 - Meromorphic functions}

We say that a complex-valued function $f$ is analytic at a point $z_{0} \in \mathbb{C}$ if there exists a convergent power series for $f$ about $z_{0}$ with positive radius of convergence. We say that $f$ is entire if it is analytic at all points in $\mathbb{C}$, and meromorphic if it is analytic at all points in $\mathbb{C}$ except for some isolated points at which it takes the value infinity, and with the property that the limit of the function at these points is infinity (there are no essential singularities). We further say $f$ is rational if it can be written in the form

$$
f(z)=\frac{P(z)}{Q(z)}
$$


where $P$ and $Q$ are polynomials with no common zeros, and $Q \not \equiv 0$. If $f$ is meromorphic and cannot be written in this form then it is said to be transcendental. We note that an entire function can only be rational if $Q^{\prime}(z) \equiv 0$, i.e. $f$ is a polynomial.

We may consider a meromorphic function on a domain to be the quotient of two analytic functions on that domain, having poles when the denominator is zero. Points $z_{0}$ where both the denominator and numerator functions have zeros are handled using the limit as $z \rightarrow z_{0}$.

\section{Examples 1.1.2}

- The function $e^{z}$ is entire, since it is analytic at all points in $\mathbb{C}$.

- The function $\frac{1}{e^{z}-1}$ is meromorphic, since it has isolated poles at integer multiples of $2 \pi i$.

- The function $e^{1 / z}$ is not meromorphic because the limit as $z$ tends to 0 is undefined.

\section{Definition 1.1.3 - The multiplicity of a zero}

Given a function $f$ which is analytic and not identically 0 in the neighbourhood of a point $z_{0} \in \mathbb{C}$ such that $f\left(z_{0}\right)=0$, we define the order or multiplicity of the zero at that point as the least $n$ such that the coefficient of $\left(z-z_{0}\right)^{n}$ in the Taylor expansion of $f$ about $z_{0}$ is non-zero. If $1 / f$ has a zero of order $n$ at $z_{0}$, we say that $f$ has a pole of order $n$ at $z_{0}$. A pole of order 1 is called a simple pole.

We may consider a zero to be a pole of negative order, and vice versa. It is quite easy to see that if at some point two functions $f$ and $g$ have poles of order $j$ and $k$ respectively, then $f g$ has a pole of order $j+k$, and $f+g$ has at most a pole of order 
$\max \{j, k\}$ (it is possible that the two poles could cancel out, for instance if $f=z^{-1}$ and $\left.g=-z^{-1}\right)$.

\section{Definitions 1.1.4 - The (unintegrated) counting function}

Given a function $f$, meromorphic in the plane, we define the (unintegrated) counting function, $n(r, f)$, to be the number of poles (counting multiplicity) of $f$ in $\bar{B}(0, r)$, the closed disc of radius $r$ centred on the origin. Thus a simple pole adds 1, a double pole adds 2 , etc. We further define $\bar{n}(r, f)$ to be the number of distinct poles of $f$ in $\bar{B}(0, r)$, that is, the number of poles of $f$ not counting multiplicity. Thus, a pole of multiplicity $k \geq 1$ adds 1 , no matter how large $k$ is.

\section{Examples 1.1.5}

- $n\left(r, e^{z}\right)=\bar{n}\left(r, e^{z}\right)=0$ since $e^{z}$ has no poles in the closed disc $\bar{B}(0, r)$.

- $n\left(r, \frac{1}{e^{z}-1}\right)=\bar{n}\left(r, \frac{1}{e^{z}-1}\right)=1+2\left\lfloor\frac{r}{2 \pi}\right\rfloor$, since the function has simple poles at integer multiples of $2 \pi i$.

- $n\left(r, \csc ^{2} z\right)=2+4\left\lfloor\frac{r}{\pi}\right\rfloor$ and $\bar{n}\left(r, \csc ^{2} z\right)=1+2\left\lfloor\frac{r}{\pi}\right\rfloor$ since $\csc ^{2} z$ has double poles at integer multiples of $\pi$.

We note that the unintegrated counting function is stepwise-increasing and, since the poles of a meromorphic function are isolated, is finite for all $r$. We now state one of the most useful tools of classical complex analysis. 


\section{Proposition 1.1.6 - The Maximum Principle}

Let the function $f$ be analytic on the bounded domain $D$ and continuous on $D \cup \partial D$. Then there exists $z_{0} \in \partial D$ such that $\left|f\left(z_{0}\right)\right| \geq|f(z)|$ for all $z \in D$.

This principle is very useful for analysing entire functions, or functions which are analytic on a bounded domain. However, if we try and apply such a principle to a meromorphic function, it breaks down. For instance, if we take $f(z)=z^{-1}$, it is clear that on the unit circle, $|f(z)|=1$, but this is smaller than $|f(0)|=\infty$. We therefore need some new method to investigate meromorphic functions. Finally in this section, we introduce the Poisson-Jensen formula.

\section{Proposition 1.1.7 - The Poisson-Jensen formula}

Let $R$ be finite and positive, $f$ be meromorphic on a domain and not identically 0 in $\bar{B}(0, R)$. Let $z=r e^{i \phi} \in B(0, R)$. Then

$$
\begin{aligned}
\log |f(z)|=\frac{1}{2 \pi} & \int_{0}^{2 \pi} \log \left|f\left(R e^{i \theta}\right)\right| \frac{R^{2}-r^{2}}{R^{2}+r^{2}-2 R r \cos (\theta-\phi)} d \theta+d \log \left|\frac{z}{R}\right|+ \\
& +\sum_{j=1}^{m} \log \left|\frac{R\left(z-a_{j}\right)}{R^{2}-\overline{a_{j}} z}\right|-\sum_{k=1}^{n} \log \left|\frac{R\left(z-b_{k}\right)}{R^{2}-\overline{b_{k}} z}\right|
\end{aligned}
$$

where $a_{1}, \ldots, a_{m}$ and $b_{1}, \ldots, b_{n}$ are respectively the zeros and poles of $f$ in $0<|z|<R$, with repetition according to multiplicity; and where the first term of the Laurent expansion of $f$, valid on some annulus centred on the origin, is the term in $z^{d}$.

\subsection{The Nevanlinna functionals}

The main tool used throughout this thesis is Nevanlinna Theory. This provides a means to analyse meromorphic functions, since classical methods such as the Maximum Principle break down when confronted with poles. The seminal work in this field is Hayman's 
Meromorphic Functions [16], where he laid out the fundamental results of Nevanlinna Theory, and applied them to problems including differential polynomials. We use Hayman's notation throughout. The results and theory presented here are taken from [16], or are easily derived from it.

\section{Definitions 1.2.1}

We first define the (Integrated) Counting Function, $N(r, f)$ :

$$
N(r, f)=\int_{0}^{r}[n(t, f)-n(0, f)] \frac{d t}{t}+n(0, f) \log r
$$

If $f$ has, counting multiplicity, $p$ poles on $|z|=q$ for some $q>0$, then these contribute $p$ to $n(t, f)-n(0, f)$ for $t \geq q$, and thus $p \log (r / q)$ to $N(r, f)$. We similarly use $\bar{n}(r, f)$ to generate $\bar{N}(r, f)$.

We define the Proximity Function, $m(r, f)$, by

$$
m(r, f)=\frac{1}{2 \pi} \int_{0}^{2 \pi} \log ^{+}\left|f\left(r e^{i \theta}\right)\right| d \theta
$$

where $\log ^{+} x=\max \{\log x, 0\}$.

We define the Nevanlinna Characteristic, $T(r, f)$ as the sum of the Proximity and Counting functions,

$$
T(r, f)=m(r, f)+N(r, f) .
$$

We note that if $f$ is an entire function it has no poles within the disc $\bar{B}(0, r)$, and so $N(r, f) \equiv 0$, and therefore $T(r, f) \equiv m(r, f)$.

We write $S(r, f)$ for any terms which are o $(T(r, f))$ as $r \rightarrow \infty$, possibly outside some 
set of finite measure.

In many cases, we will be concerned with a function of the form $1 /(f-a)$ for some $a \in \mathbb{C}$. As a form of shorthand, we may write the Characteristic of this function as $T(r, a, f)$. The Proximity and Counting functions may be written similarly.

We now state some basic properties of these functionals.

\section{Lemma 1.2.2}

Let $f$ and $g$ be meromorphic and non-constant in the plane, and let $k \in \mathbb{N}$. Then the following are true:

$$
\begin{aligned}
\bar{N}(r, f) & \leq N(r, f) \\
N\left(r, f^{k}\right) & =k N(r, f) \\
m\left(r, f^{k}\right) & =k m(r, f) \\
T\left(r, f^{k}\right) & =k T(r, f) \\
N(r, f+g) & \leq N(r, f)+N(r, g) \\
m(r, f+g) & \leq m(r, f)+m(r, g)+\log 2 \\
T(r, f+g) & \leq T(r, f)+T(r, g)+\log 2 \\
N(r, f g) & \leq N(r, f)+N(r, g) \\
m(r, f g) & \leq m(r, f)+m(r, g) \\
T(r, f g) & \leq T(r, f)+T(r, g)
\end{aligned}
$$

These are all quite elementary from the definitions of the functionals. We now compare $T(r, f)$ and the maximum modulus function. 


\section{Lemma 1.2.3}

Let $0<r<R$, and let $f$ be analytic on the disc $\bar{B}(0, R)$, and define $M(r, f)$, the maximum modulus function, by

$$
M(r, f)=\max \{|f(z)|: z \in \bar{B}(0, r)\} .
$$

Note that, by Proposition 1.1.6, this maximal value is taken on the circle of radius $r$, centred on the origin. Then

$$
\begin{array}{r}
T(r, f) \leq \log ^{+} M(r, f) \\
\log M(r, f) \leq\left(\frac{R+r}{R-r}\right) T(R, f) .
\end{array}
$$

The first of these inequalities follows from the fact that $N(r, f)=0$, and the second from an application of the Poisson-Jensen formula (1.1.1).

\subsection{The Fundamental Theorems}

We now move on to some basic theorems of Nevanlinna Theory, which many of our later results reference. We start with one of the most important theorems, which follows from taking the limit of the Poisson-Jensen formula (1.1.1) as $z \rightarrow 0$.

\section{Proposition 1.3.1 - The First Fundamental Theorem}

Let $f$ be a non-constant meromorphic function, and $a \in \mathbb{C}$. Then, as $r \rightarrow \infty$,

$$
T\left(r, \frac{1}{f-a}\right)=T(r, f)+O(1)
$$

In particular, this allows us to say that $T(r, f)=T(r, 1 / f)+S(r, f)$, which is exceedingly useful. The proof of this is by the Poisson-Jensen formula (1.1.1). We now make a note about the characteristic of a rational function. 


\section{Lemma 1.3.2}

$T(r, f)=O(\log r)$ if and only if $f$ is a rational function.

This lemma is very useful in the later sections of this work. We now define the order of a function.

\section{Definition 1.3.3}

Let $f$ be meromorphic in the plane. Then we define the order $\rho(f)$ by

$$
\rho(f)=\limsup _{r \rightarrow \infty} \frac{\log ^{+} T(r, f)}{\log r}
$$

We say that $f$ has finite order if $\rho(f)<\infty$, or equivalently $T(r, f)=O\left(r^{\rho}\right)$. In particular, a rational function has order 0.

\section{Example 1.3.4}

$T\left(r, e^{z}\right)=r$ for $a \in \mathbb{C}$, and thus $e^{z}$ has order 1 . Further, for $n \in \mathbb{N}, T\left(r, e^{z^{n}}\right)=r^{n}$, and thus $e^{z^{n}}$ has order $n$. However, $T\left(r, e^{e^{z}}\right)=O\left(e^{z}\right)$, and thus $e^{e^{z}}$ has infinite order.

\section{Lemma 1.3.5 - The Lemma of the Logarithmic Derivative}

Let $f$ be non-constant and meromorphic in the plane. Then there are positive constants $c_{j}$ such that as $r \rightarrow \infty$ outside some set of finite measure,

$$
m\left(r, \frac{f^{\prime}}{f}\right) \leq c_{1} \log r+c_{2} \log T(r, f)
$$

If $f$ is of finite order, then $m\left(r, f^{\prime} / f\right)=O(\log r)$. In general, we simply say that, for transcendental $f, m\left(r, f^{\prime} / f\right)=S(r, f)$. Also note that since $m(r, f g) \leq m(r, f)+m(r, g)$ for functions $f$ and $g$, we can further say that, by induction,

$$
m\left(r, \frac{f^{(k)}}{f}\right) \leq m\left(r, \frac{f^{(k)}}{f^{(k-1)}}\right)+m\left(r, \frac{f^{(k-1)}}{f^{(k-2)}}\right)+\ldots+m\left(r, \frac{f^{\prime}}{f}\right) \leq c_{1} \log r+c_{2} \log T(r, f) .
$$




\section{Lemma 1.3.6}

Let $f$ be non-constant and meromorphic in the plane. Then

$$
T\left(r, f^{(k)}\right) \leq T(r, f)+k \bar{N}(r, f)+S(r, f) .
$$

This follows from the Lemma of the Logarithmic Derivative (Lemma 1.3.5) and the properties of the characteristic of two functions from Lemma 1.2.2. We now state what is called the Second Fundamental Theorem.

\section{Proposition 1.3.7 - The Second Fundamental Theorem}

Let $f$ be meromorphic in the plane. Then given any $k$ distinct values $b_{j}$ in $\mathbb{C}^{*}:=$ $\mathbb{C} \cup\{\infty\}$, we have that

$$
(k-2) T(r, f) \leq \sum_{j=1}^{k} \bar{N}\left(r, b_{j}, f\right)+S(r, f),
$$

which follows from the inequality

$$
\sum_{j=1}^{k} m\left(r, b_{j}, f\right) \leq 2 T(r, f)-N_{1}(r, f)+S(r, f),
$$

where $N_{1}(r, f)=N(r, f)-\bar{N}(r, f)+N\left(r, 1 / f^{\prime}\right)$.

This theorem allows us to easily prove Picard's Theorem - suppose $f$ is transcendental and entire, and takes two finite values $b_{1}$ and $b_{2}$ only finitely often. Let $k=3$ and $b_{3}=\infty$. Then (1.3.3) gives $T(r, f) \leq O(\log r)+S(r, f)$, which by Lemma 1.3.2 gives that $f$ is rational, a contradiction. 


\section{Definition 1.3.8 - The Nevanlinna Deficiency}

Let $f$ be meromorphic in the plane, and $a \in \mathbb{C}^{*}$. We define the Nevanlinna Deficiency $\delta$ of the point a by

$$
\delta(a, f)=1-\limsup _{r \rightarrow \infty} \frac{N(r, a, f)}{T(r, f)}=\liminf _{r \rightarrow \infty} \frac{m(r, a, f)}{T(r, f)} .
$$

This describes how infrequently the value a is taken by $f$. By the First Fundamental Theorem, it is clear that $\delta(a, f) \in[0,1]$ - for a transcendental function $f$, any value which is only taken finitely often would have a deficiency of 1. By the Second Fundamental Theorem (1.3.4), we can see that

$$
\sum_{a \in \mathbb{C}^{*}} \delta(a, f) \leq 2
$$

This is called the Defect Relation.

\section{Examples 1.3.9}

- $\delta(a, z)=0$ for any $a \in \mathbb{C}$, since if $a=0$ then $N(r, 0, z)=\log r$, and if $a \neq 0$ and $r>|a|$ then $N(r, a, z)=\log (r /|a|)=\log r-\log |a| \rightarrow \log r$ as $r \rightarrow \infty$.

- $\delta\left(0, e^{z}\right)=1$ because $e^{z}$ omits 0 , and thus $N\left(r, 0, e^{z}\right) \equiv 0$. Further, since $N(r, f) \equiv$ $0, \delta\left(\infty, e^{z}\right)=1$, and thus by (1.3.6) $\delta\left(a, e^{z}\right)=1$ for any non-zero $a \in \mathbb{C}$.

- A function can take a value infinitely often but still have a deficiency of 1 at that point. For instance if we take $f=e^{z^{2}} \tan z, \delta(0, f)=1$ since $N(r, 0, f)=$ $O(T(r, \tan z))=O(r)$, but $T(r, f) \geq T\left(r, e^{z^{2}}\right)=O\left(r^{2}\right)$.

- It is also possible to have functions take values with deficiency in the open interval $(0,1)$, but such examples are difficult to construct and beyond the scope of this work. 


\subsection{Differential polynomials}

Most of the results in this thesis are concerned with the analysis of differential polynomials. Differential polynomials have been extensively investigated over the years, beginning with Chapter 3 of [16]. We will now give a brief summary of their properties.

\section{Definitions 1.4.1}

Let $f$ be a function, meromorphic in the plane, and define $M_{j}$ by

$$
M_{j}[f]=f^{\mu_{0, j}}\left(f^{\prime}\right)^{\mu_{1, j}} \ldots\left(f^{(q)}\right)^{\mu_{q, j}},
$$

where the $\mu_{k, j}$ are non-negative integers. We call $M_{j}$ a differential monomial in $f$. We say that $M_{j}$ has degree

$$
\gamma_{j}=\mu_{0, j}+\ldots+\mu_{q, j}
$$

and weight

$$
\Gamma_{j}=\mu_{0, j}+2 \mu_{1, j}+\ldots+(q+1) \mu_{q, j}
$$

The weight of a monomial $M_{j}[f]$ is the order of the pole such a monomial would take if $f$ were to have a simple pole at a point. We further define a differential polynomial $P[f]$ as a sum of differential monomials with suitable functions as coefficients. We say that $P$ has degree $\gamma=\max \left\{\gamma_{j}\right\}$, and weight $\Gamma=\max \left\{\Gamma_{j}\right\}$. We call a differential polynomial linear if $\gamma \leq 1$, and non-linear if $\gamma>1$. We further say that a differential polynomial is homogeneous if all terms have equal degree.

Exactly which functions are suitable coefficients is dependent upon the specific use, and will be stated explicitly later. General classes of suitable functions include constants, rational functions and functions $c_{j}$ such that $T\left(r, c_{j}\right)=S(r, f)$. 


\section{Example 1.4.2}

Let $F=f^{2}\left(f^{\prime \prime}\right)^{3}+f^{\prime} f^{(8)}+\left(f^{\prime \prime \prime}\right)^{3}$ be a differential polynomial in a meromorphic function $f$. Then $F$ has degree

$$
\gamma=\max \{5,2,3\}=5
$$

and weight

$$
\Gamma=\max \{(2 * 1+3 * 3=11),(1 * 2+1 * 9=11),(3 * 4=12)\}=12 .
$$

Finally, we introduce a very useful lemma which allows us to find the general form of a function given a certain condition.

\section{Lemma 1.4.3}

If $f^{\prime} / f$ is a rational function, then $f=R e^{P}$, where $R$ is a rational function and $P$ is a polynomial.

This proof of this lemma uses partial fractions, and is standard knowledge. 


\section{Chapter 2}

\section{Pairs of non-homogeneous linear}

\section{differential polynomials}

In [21], Langley proved a result concerning the zeros of pairs of (possibly nonhomogeneous) linear differential polynomials in a meromorphic function. In this chapter we generalise this result by relaxing Langley's assumption on the frequency of zeros (counting multiplicity), and further prove some results based on restricting the order of the differential operators. This work (with the exception of Theorem 2.2.4) was published in Computational Methods and Function Theory [5].

\section{$2.1 \quad$ Introduction}

Let $f$ be a non-constant meromorphic function in the plane. Throughout this section, we use the convention that $c_{s}$ is a "small function" - i.e. a function such that $T\left(r, c_{s}\right)=$ $S(r, f)$. We further define a linear differential polynomial $\psi$ in $f$ by

$$
\psi=\sum_{s=0}^{t} c_{s} f^{(s)} \text {. }
$$


We begin with a result of Milloux from [16], which may be viewed as a counterpart of Nevanlinna's Second Fundamental Theorem.

\section{Proposition 2.1.1}

Let $f$ be meromorphic and non-constant in the plane, and $\psi$ as defined by (2.1.1) also be non-constant. Then,

$$
T(r, f)<\bar{N}(r, f)+N\left(r, \frac{1}{f}\right)+\bar{N}\left(r, \frac{1}{\psi-1}\right)-N_{0}\left(r, \frac{1}{\psi^{\prime}}\right)+S(r, f),
$$

where $N_{0}\left(r, 1 / \psi^{\prime}\right)$ counts only zeros of $\psi^{\prime}$ which are not multiple 1-points of $\psi$.

Hayman showed in [16] that for $\psi=f^{(k)}$ with $k \geq 1$, a version of (2.1.2) holds without the $\bar{N}(r, f)$ term and, in particular, that if $f$ omits 0 then $f^{(k)}$ must take every finite non-zero value. This was subsequently extended by Bergweiler and Langley in [4] to linear differential polynomials in $f$, subject to conditions on the coefficients $c_{j}$.

It is possible to have both $f$ and $f^{(k)}$ omitting 0 , but it was shown in [14] and [19] that if $f$ and $f^{(k)}$ have only finitely many zeros for some $k \geq 2$ then $f=R e^{P}$ where $R$ is a rational function and $P$ a polynomial. The following result from [15] addresses the case where two homogeneous linear differential polynomials have few zeros.

\section{Proposition 2.1.2}

Let $f$ be meromorphic and non-constant in the plane, and let $L_{1}$ and $L_{2}$ be homogeneous linear differential operators, with coefficients which are rational functions and leading terms $\frac{d^{k}}{d z^{k}}$ and $\frac{d^{n}}{d z^{n}}$ respectively, with $k \geq n \geq 1$. Let $F=L_{1}(f)$ and $G=L_{2}(f)$, assume that

$$
\bar{N}\left(r, \frac{1}{F}\right)+\bar{N}\left(r, \frac{1}{G}\right)=O\left(\log ^{+} T\left(r, \frac{f^{\prime}}{f}\right)+\log r\right)
$$


as $r \rightarrow \infty$ outside a set of finite measure and further assume that the equations $L_{1}(\omega)=0$ and $L_{2}(\omega)=0$ have no non-trivial common (local) solutions, so that in particular $L_{1}$ and $L_{2}$ are not the same.

Then $f$ has finite order and finitely many zeros and $f^{\prime} / f$ has a representation

$$
\frac{f^{\prime}(z)}{f(z)}=Y(z)+\frac{P[Q(z)+\log R(z)]\left(Q^{\prime}(z)+R^{\prime}(z) / R(z)\right)}{R(z) e^{Q(z)}-1}
$$

in which $Y$ and $R$ are rational functions and $P$ and $Q$ are polynomials, and at least one of $P$ and $R$ is constant.

We now make some definitions which will be used throughout the rest of this chapter.

\section{Definitions 2.1.3}

Let $L$ and $M$ be linear differential operators of positive order $k$ and $n$ respectively, with

$$
L=\frac{d^{k}}{d z^{k}}+\sum_{j=0}^{k-1} a_{j} \frac{d^{j}}{d z^{j}}, \quad M=\frac{d^{n}}{d z^{n}}+\sum_{j=0}^{n-1} b_{j} \frac{d^{j}}{d z^{j}},
$$

where the coefficients $a_{j}, b_{j}$ are rational functions, and where the equations $L(\omega)=0$ and $M(\omega)=0$ have no common non-trivial (local) solutions (i.e. other than the solution which is identically zero). Then by lemmas from [15], there exist linear differential operators $P, Q, U, V$ and $Y$ with coefficients which are rational functions in the $a_{j}, b_{j}$ and their derivatives such that

$$
P(L)+Q(M)=1, \quad Y=U(L)=V(M),
$$

where 1 is the identity operator, and $U, V, Y$, have order $n, k, n+k$, and leading terms $\frac{d^{n}}{d z^{n}}, \frac{d^{k}}{d z^{k}}, \frac{d^{n+k}}{d z^{n+k}}$ respectively. The (local) solution space of $Y(\omega)=0$ is the direct sum of the (local) solution spaces of the equations $M(\omega)=0$ and $L(\omega)=0$. The parentheses 
in (2.1.4) denote composition. We now define linear differential polynomials $F$ and $G$ by

$$
F=L(f)+a, \quad G=M(f)+b,
$$

where $f$ is meromorphic in the plane, and $a, b$ are rational functions and we assume that $F \not \equiv 0, G \not \equiv 0$. We define a rational function $c$ by

$$
c=P(a)+Q(b)
$$

and set

$$
g=f+c=P(F)+Q(G) .
$$

Now, from these definitions, we see that

$$
F=L(f)+a=L(g)+a-L(c), \quad G=M(f)+b=M(g)+b-M(c) .
$$

Furthermore,

$$
U(F)=V(G)+d, \quad d=U(a)-V(b)
$$

where $d$ is a rational function.

Finally, let $\Omega$ be a non-empty simply-connected domain on which the functions $a, b$, and the coefficients $a_{j}, b_{j}$ are analytic. Let the linearly independent solutions of $L(\omega)=0$ and $M(\omega)=0$ be, respectively, $u_{1}, \ldots, u_{k}$ and $v_{1}, \ldots, v_{n}$, and let $u$ and $v$ be solutions of $L(\omega)=a$ and $M(\omega)=b$ respectively

We now state Langley's result from [21], which provides our springboard for the results which follow. 


\section{Proposition 2.1.4}

Let the function $f$ be transcendental and meromorphic in the plane, and suppose that Definitions 2.1.3 hold. Assume that

$$
N\left(r, \frac{1}{F}\right)+N\left(r, \frac{1}{G}\right)=S(r, f) .
$$

Then at least one of the following holds:

1. $F=L(g)$ and $G=M(g)$;

2. $f$ has a representation $f=R\left(u_{1}, \ldots, u_{k}, v_{1}, \ldots, v_{n}, u, v\right)$, where $R$ is a rational function in $k+n+2$ variables.

\subsection{Results}

We begin by stating our results, and will then give the proofs later. Our first result weakens the assumption (2.1.10) in terms of $T(r, f)$.

\section{Theorem 2.2.1}

Let the function $f$ be transcendental and meromorphic in the plane, and let Definitions 2.1.3 hold. Assume that $\gamma_{1} \geq 0, \gamma_{2} \geq 0$ and that

$$
N\left(r, \frac{1}{F}\right) \leq \gamma_{1} T(r, f)+S(r, f), \quad N\left(r, \frac{1}{G}\right) \leq \gamma_{2} T(r, f)+S(r, f) .
$$

Further define

$$
\gamma_{0}=\max \left\{\gamma_{1}, \gamma_{2}\right\}, \quad \gamma_{3}=\gamma_{1}+\gamma_{2}
$$

Then at least one of the following holds:

1. $F=L(g)$ and $G=M(g)$; 
2. $f$ has a representation $f=R\left(u_{1}, \ldots, u_{k}, v_{1}, \ldots, v_{n}, u, v\right)$, where $R$ is a rational function in $k+n+2$ variables.

3. the $\gamma_{j}$ satisfy

$$
\frac{k+n}{k+n+1} \leq 2 \gamma_{3}+\gamma_{0}+\frac{2 \gamma_{3}+1}{k+n}
$$

Remark: We note here that conclusions 1 and 2 are as in Proposition 2.1.4, and that (2.2.3) cannot hold if $\gamma_{1}=\gamma_{2}=0$, so that Theorem 2.2.1 reduces to Proposition 2.1.4 in this case. Further, if $\gamma_{3} \geq 0.4$ then $\gamma_{0} \geq 0.2$, and the inequality (2.2.3) will always hold, in which case the conclusions of Proposition 2.1.4 need not hold.

Our second result shows that if $k=n$ then $N$ can be replaced by $\bar{N}$ in the hypothesis (2.1.10) of Proposition 2.1.4.

\section{Theorem 2.2.2}

Let $f$ be transcendental and meromorphic in the plane, let Definitions 2.1.3 hold with $k=n$, and assume that

$$
\bar{N}\left(r, \frac{1}{F}\right)+\bar{N}\left(r, \frac{1}{G}\right)=S(r, f) .
$$

Then at least one of the following must hold:

1. $F=L(g)$ and $G=M(g)$;

2. $f$ has a representation $f=R\left(u_{1}, \ldots, u_{n}, v_{1}, \ldots, v_{n}, u, v\right)$, where $R$ is a rational function in $2 n+2$ variables.

The next result also replaces (2.1.10) by (2.2.4). The last two results are more minor, but we present them for completeness. 


\section{Theorem 2.2.3}

Let $f$ be transcendental and meromorphic in the plane, and let Definitions 2.1 .3 hold with $b \equiv 0$ and $a-L(c) \not \equiv 0$. Suppose that (2.2.4) holds and suppose further that

$$
n>k+2 \text {. }
$$

Then at least one of the following holds:

1. $F=L(g)$ and $G=M(g)$;

2. $f$ has a representation $f=R\left(u_{1}, \ldots, u_{k}, v_{1}, \ldots, v_{n}, u, v\right)$, where $R$ is a rational function in $k+n+2$ variables.

\section{Theorem 2.2.4}

Let $f$ be transcendental and meromorphic in the plane, and let Definitions 2.1.3 and (2.1.10) hold.

If $a-L(c) \not \equiv 0$, then at least one of the following holds:

1. $f$ has finitely many poles;

2. $n \leq 2$;

3. $f$ has a rational representation $f=R\left(f_{1}, \ldots, f_{n+1}\right)$ where the $f_{j}$ are (local) solutions to $M(\omega)=d_{j} b$ and each $d_{j}$ is a constant.

If $b-M(c) \not \equiv 0$, then at least one of the following holds:

1. $f$ has finitely many poles;

2. $k \leq 2$;

3. $f$ has a rational representation $f=R\left(f_{1}, \ldots, f_{k+1}\right)$ where the $f_{j}$ are (local) solutions to $L(\omega)=d_{j}$ a and each $d_{j}$ is a constant. 


\subsection{Preliminary lemmas}

In this section we state and then refine a lemma from [21] which will be very useful in our proofs. The case where $A \equiv 0$ is treated in [13] and [30].

\section{Lemma 2.3.1 [21]}

Let $\delta$ be a positive real number, and let the function $h$ be transcendental and meromorphic in the plane. Let $p$ be a positive integer, and $c_{0}, c_{1}, \ldots, c_{p-1}$ and $A$ be rational functions. Set

$$
\begin{array}{r}
Q_{p}=\frac{d^{p}}{d z^{p}}+\sum_{j=0}^{p-1} c_{j} \frac{d^{j}}{d z^{j}}, \\
H=Q_{p}(h)+A .
\end{array}
$$

Then at least one of the following conditions holds:

(i) we have

$$
p \bar{N}(r, h) \leq N\left(r, \frac{1}{H}\right)+(1+\delta) N(r, h)+S(r, h)
$$

(ii) $h$ has a representation

$$
h=R\left(h_{1}, \ldots, h_{p+1}\right)
$$

where $R$ is a rational function in $p+1$ variables and each $h_{j}$ is a (local) solution of

$$
Q_{p}(\omega)=d_{j} A,
$$

with $d_{j}$ a constant.

We now present a refinement of Langley's result. 


\section{Lemma 2.3.2}

Let $h$ in Lemma 2.3.1 be such that (ii) does not hold. Then:

$$
p \bar{N}(r, h) \leq N\left(r, \frac{1}{H}\right)+N(r, h)+S(r, h) .
$$

Proof:

Assuming that Lemma 2.3.1 (ii) does not hold, then (i) must hold for any $\delta>0$. In particular, for all $n \in \mathbb{N}$,

$$
\begin{aligned}
p \bar{N}(r, h) & \leq N\left(r, \frac{1}{H}\right)+\left(1+\frac{1}{2 n}\right) N(r, h)+S(r, h) \\
& \leq N\left(r, \frac{1}{H}\right)+N(r, h)+\frac{1}{n} T(r, h)
\end{aligned}
$$

for all $r \geq 1$ outside a set $E_{n}$ of finite measure. Now, take a sequence $\left(r_{n}\right)$ such that $F_{n}=E_{n} \cap\left[r_{n}, \infty\right)$ has measure at most $n^{-2}$, with $r_{n} \geq r_{n-1}+1$, and $r_{1} \geq 1$. Then $r_{n} \rightarrow \infty$. Let

$$
F_{0}=\bigcup_{n=1}^{\infty} F_{n} .
$$

Then $F_{0}$ has measure at most $1+2^{-2}+3^{-2}+\ldots<\infty$. Now let $r \notin F_{0}$ be large, and $r_{m}$ the largest member of $\left(r_{n}\right)$ which is not greater than $r$. Then $m$ is large and $r \in\left[r_{m}, \infty\right)$. However, $r \notin E_{m}$, so

$$
p \bar{N}(r, h) \leq N\left(r, \frac{1}{H}\right)+N(r, h)+\frac{1}{m} T(r, h) .
$$

Thus, as $r \rightarrow \infty$ with $r \notin F_{0}$ (and thus $m \rightarrow \infty$ ),

$$
p \bar{N}(r, h) \leq N\left(r, \frac{1}{H}\right)+N(r, h)+o(T(r, h))
$$

which leads immediately to (2.3.4) by our definition of $S(r, h)$. 


\subsection{Proofs of the theorems}

\subsubsection{Initial steps}

Assume that $f$ is transcendental meromorphic in the plane, and that the Definitions 2.1.3 hold. We state and prove several lemmas.

\section{Lemma 2.4.1}

If either $U(F)$ or $V(G)$ is a rational function then $f$ has finitely many poles and at least one of the following must hold:

1. The following inequality holds:

$$
\begin{aligned}
T(r, f) & \leq N\left(r, \frac{1}{F}\right)+N\left(r, \frac{1}{G}\right)+S(r, f) \\
& \leq n \bar{N}\left(r, \frac{1}{F}\right)+k \bar{N}\left(r, \frac{1}{G}\right)+S(r, f) .
\end{aligned}
$$

2. $f$ has a representation $f=R\left(u_{1}, \ldots, u_{k}, v_{1}, \ldots, v_{n}, u, v\right)$, where $R$ is a rational function in $k+n+2$ variables, and the $u_{j}, v_{j}, u$ and $v$ are as in Definitions 2.1.3.

Proof:

Assume without loss of generality that $U(F)$ is rational; then by $(2.1 .9)$ so is $V(G)$. Assume that neither vanishes identically. Then $F$ and $G$ each solve a non-homogeneous linear differential equation with rational coefficients, and

$$
\frac{U(F)}{F}=\frac{F^{(n)}}{F}+\ldots=\frac{R_{0}}{F}
$$

where $R_{0} \not \equiv 0$ is a rational function. Thus, by Lemmas 1.2.2, 1.3.2 and the Lemma of 
the Logarithmic Derivative (Lemma 1.3.5),

$$
\begin{aligned}
m\left(r, \frac{1}{F}\right) & \leq m\left(r, \frac{U(F)}{F}\right)+m\left(r, \frac{1}{U(F)}\right)+O(1) \\
& \leq m\left(r, \frac{F^{(n)}}{F}\right)+\ldots m\left(r, c_{1} \frac{F^{\prime}}{F}\right)+m\left(r, c_{0}\right)+T\left(r, R_{0}\right)+O(1) \\
& \leq S(r, f)
\end{aligned}
$$

and similarly for $G$, where the $c_{j}$ are the coefficients of $U$. Thus, by the First Fundamental Theorem,

$$
\begin{aligned}
T(r, F)+T(r, G) & \leq T\left(r, \frac{1}{F}\right)+T\left(r, \frac{1}{G}\right)+S(r, f) \\
& \leq N\left(r, \frac{1}{F}\right)+N\left(r, \frac{1}{G}\right)+S(r, f)
\end{aligned}
$$

Now, we have that

$$
P(F)=\sum_{j=0}^{p} \alpha_{j} F^{(j)},
$$

where $\alpha_{j}$ are rational coefficients, and thus, by the Lemma of the Logarithmic Derivative and (1.2.12),

$$
m(r, P(F)) \leq m(r, F)+m\left(r, \frac{P(F)}{F}\right)=m(r, F)+S(r, f)
$$

Moreover, as $U(F)$ is rational, $F$ has only finitely many poles, and so does $f$ since, apart from finitely many exceptions due to the coefficients in $L$, a pole of $f$ must generate a pole of $F$. Thus,

$$
N(r, P(F))=O(\log r)
$$

and similarly for $Q(G)$. Thus, by (1.2.10) and (2.1.7),

$$
\begin{aligned}
T(r, f) & \leq T(r, g)+S(r, f) \\
& \leq T(r, F)+T(r, G)+S(r, f),
\end{aligned}
$$

to which we apply (2.4.3) to obtain (2.4.1). Now, a zero $z_{0}$ of $F$ of multiplicity $m>n$ with $z_{0}$ large is a zero of $U(F)$ of multiplicity at least $m-n$, but this is impossible since 
$U(F)$ is rational. Thus

$$
N\left(r, \frac{1}{F}\right) \leq n \bar{N}\left(r, \frac{1}{F}\right)+S(r, f)
$$

and by the same method,

$$
N\left(r, \frac{1}{G}\right) \leq k \bar{N}\left(r, \frac{1}{G}\right)+S(r, f)
$$

We then apply these to (2.4.1) to obtain (2.4.2).

Now assume without loss of generality that $U(F) \equiv 0$. Then by (2.1.4) and (2.1.5), $0=U(F)=Y(f)+U(a)$ so that with the $u_{j}, v_{j}$ and $u$ as defined, $f+u$ solves $Y(\omega)=0$ and is a linear combination of $u_{1}, \ldots, u_{k}, v_{1}, \ldots, v_{n}$ on $\Omega$. Thus $f$ has a representation as asserted.

It is clear from (2.1.8) that if $a-L(c)$ and $b-M(c)$ both vanish identically, then $F=L(g)$ and $G=M(g)$ are satisfied. Hence we assume in the next lemma that at least one of $B=a-L(c)$ and $C=b-M(c)$ does not vanish identically.

\section{Lemma 2.4 .2}

Assume that both $U(F)$ and $V(G)$ are transcendental. Then if $a-L(c) \not \equiv 0$

$$
T(r, f) \leq N\left(r, \frac{1}{g}\right)+\bar{N}(r, f)+\bar{N}\left(r, \frac{1}{F}\right)+S(r, f),
$$

and

$$
T(r, f) \leq(k+1) \bar{N}\left(r, \frac{1}{g}\right)+\bar{N}(r, f)+\bar{N}\left(r, \frac{1}{F}\right)+S(r, f) .
$$


If $b-M(c) \not \equiv 0$ then

$$
T(r, f) \leq N\left(r, \frac{1}{g}\right)+\bar{N}(r, f)+\bar{N}\left(r, \frac{1}{G}\right)+S(r, f),
$$

and

$$
T(r, f) \leq(n+1) \bar{N}\left(r, \frac{1}{g}\right)+\bar{N}(r, f)+\bar{N}\left(r, \frac{1}{G}\right)+S(r, f)
$$

Proof:

Assume that $B=a-L(c) \not \equiv 0$. We aim to apply Milloux's result (2.1.2) to $F=$ $L(f)+a$, but to do so we must rearrange this to take account of $a$ being nonconstant. To this end, we take $f+c=g=B g^{*}$ and $F=L(g)+B=B\left(L^{*}\left(g^{*}\right)+1\right)$, where $B$ is a rational function and $L^{*}$ is a linear differential operator. Hence

$$
\bar{N}\left(r, \frac{1}{L^{*}\left(g^{*}\right)+1}\right)=\bar{N}\left(r, \frac{1}{F}\right)+S(r, f),
$$

and

$$
T(r, f)=T\left(r, g^{*}\right)+S(r, f), \quad \bar{N}(r, f)=\bar{N}(r, g)+S(r, f)=\bar{N}\left(r, g^{*}\right)+S(r, f) .
$$

Since $U(F)$ is transcendental, so is $F$, and thus $\left(L^{*}\left(g^{*}\right)+1\right)^{\prime} \not \equiv 0$, and so we may apply Milloux's result (2.1.2) to $g^{*}$, giving

$$
T\left(r, g^{*}\right) \leq \bar{N}\left(r, g^{*}\right)+N\left(r, \frac{1}{g^{*}}\right)+\bar{N}\left(r, \frac{1}{L^{*}\left(g^{*}\right)+1}\right)-N_{0}\left(r, \frac{1}{\left(L^{*}\left(g^{*}\right)\right)^{\prime}}\right)+S\left(r, g^{*}\right),
$$

where $N_{0}$ counts the zeros of $\left(L^{*}\left(g^{*}\right)\right)^{\prime}$ which are not also zeros of $L^{*}\left(g^{*}\right)+1$. Now, a zero $z_{0}$ of $g^{*}$ of multiplicity $p$ with $z_{0}$ large contributes $p$ to $n\left(r, 1 / g^{*}\right)$ and at least $\max \{0, p-k-1\}$ to $n_{0}\left(r, 1 /\left(L^{*}\left(g^{*}\right)\right)^{\prime}\right)$, and hence at most $\min \{p, k+1\} \leq k+1$ to $n\left(r, 1 / g^{*}\right)-n_{0}\left(r, 1 /\left(L^{*}\left(g^{*}\right)\right)^{\prime}\right)$. Hence, we can rewrite (2.4.10) as

$$
T\left(r, g^{*}\right) \leq \bar{N}\left(r, g^{*}\right)+(k+1) \bar{N}\left(r, \frac{1}{g^{*}}\right)+\bar{N}\left(r, \frac{1}{L^{*}\left(g^{*}\right)+1}\right)+S\left(r, g^{*}\right),
$$


and thus, by application of (2.4.8) and (2.4.9), we obtain (2.4.5). Now, returning to (2.4.10), we may discard the $N_{0}$ term and again apply (2.4.8) and (2.4.9) to give (2.4.4).

We follow similar steps to obtain (2.4.6) and (2.4.7).

QED

\section{Lemma 2.4 .3}

Assume that $U(F)$ and $V(G)$ are transcendental. If $d=U(F)-V(G) \not \equiv 0$, then

$$
N\left(r, \frac{1}{g}\right)+N\left(r, \frac{1}{H}\right) \leq \bar{N}(r, f)+2 N\left(r, \frac{1}{F}\right)+2 N\left(r, \frac{1}{G}\right)+S(r, f)
$$

and

$$
\bar{N}\left(r, \frac{1}{g}\right)+\bar{N}\left(r, \frac{1}{H}\right) \leq \bar{N}(r, f)+A_{1}\left(\bar{N}\left(r, \frac{1}{F}\right)+\bar{N}\left(r, \frac{1}{G}\right)\right)+S(r, f)
$$

where $A_{1}$ is a positive constant and

$$
H=\left(\frac{d}{d z}-\frac{d^{\prime}}{d}\right)(U(F)) \not \equiv 0
$$

If $d \equiv 0$, then

$$
N\left(r, \frac{1}{g}\right)+N\left(r, \frac{1}{H}\right) \leq 2 N\left(r, \frac{1}{F}\right)+2 N\left(r, \frac{1}{G}\right)+S(r, f),
$$

and

$$
\bar{N}\left(r, \frac{1}{g}\right)+\bar{N}\left(r, \frac{1}{H}\right) \leq A_{2}\left(\bar{N}\left(r, \frac{1}{F}\right)+\bar{N}\left(r, \frac{1}{G}\right)\right)+S(r, f),
$$

where $A_{2}$ is a positive constant and

$$
H=U(F) \not \equiv 0
$$

Proof:

Assume first that $d=U(F)-V(G) \not \equiv 0$. We define linear differential operators $\widetilde{U}$ and $\widetilde{V}$ by

$$
\widetilde{U}=\left(\frac{d}{d z}-\frac{d^{\prime}}{d}\right)(U), \quad \widetilde{V}=\left(\frac{d}{d z}-\frac{d^{\prime}}{d}\right)(V)
$$


and thus by (2.4.13) and the definition of $d$, we have $H=\widetilde{U}(F)=\widetilde{V}(G)$. If $H \equiv 0$, then by the previous two equations, there exist constants $\mu, \nu$ such that $U(F)=\mu d$ and $V(G)=\nu d$, which, since $d$ is rational by (2.1.9), contradicts our assumption that $U(F)$ and $V(G)$ are transcendental. Thus, $H \not \equiv 0$. Set

$$
\phi=\frac{g H}{F G}=\frac{P(F) \widetilde{V}(G)}{F G}+\frac{Q(G) \widetilde{U}(F)}{F G},
$$

using (2.1.7) and (2.4.13). Since $P, Q, \widetilde{U}$ and $\widetilde{V}$ are linear differential operators with rational functions as coefficients, by the Lemma of the Logarithmic Derivative (1.3.2), $m(r, \phi)=S(r, f)$. We now turn to $N(r, \phi)$. Suppose $f$ has a pole of multiplicity $m$ at some point $z_{0}$ with $z_{0}$ large. Then $g, F, G$ and $H$ have poles at $z_{0}$ with multiplicities $m, m+k, m+n$ and $m+n+k+1$ respectively, and so $\phi$ has a simple pole at $z_{0}$. Thus, considering also the poles generated by zeros of $F$ and $G$,

$$
T(r, \phi) \leq \bar{N}(r, f)+N\left(r, \frac{1}{F}\right)+N\left(r, \frac{1}{G}\right)+S(r, f) .
$$

Writing $1 / g H=1 / \phi F G$ and using (2.4.19) leads to, since $g$ has only finitely many poles at zeros of $H$ (and vice versa),

$$
\begin{aligned}
N\left(r, \frac{1}{g}\right)+N\left(r, \frac{1}{H}\right) & \leq N\left(r, \frac{1}{g H}\right)+S(r, f) \\
& =N\left(r, \frac{1}{\phi F G}\right)+S(r, f) \\
& \leq N\left(r, \frac{1}{\phi}\right)+N\left(r, \frac{1}{F}\right)+N\left(r, \frac{1}{G}\right)+S(r, f) \\
& \leq T(r, \phi)+N\left(r, \frac{1}{F}\right)+N\left(r, \frac{1}{G}\right)+S(r, f), \quad
\end{aligned}
$$

from which (2.4.11) follows from substitution of (2.4.19).

Since a zero of $F($ respectively $G)$ gives at most a pole of $F^{(j)} / F\left(\right.$ respectively $\left.G^{(j)} / G\right)$ of multiplicity $j$, we obtain, for some $\widetilde{A_{1}}>0$,

$$
T(r, \phi) \leq \bar{N}(r, f)+\widetilde{A_{1}}\left(\bar{N}\left(r, \frac{1}{F}\right)+\bar{N}\left(r, \frac{1}{G}\right)\right)+S(r, f)
$$


Again, as $g$ has only finitely many poles at zeros of $H$ (and vice versa),

$$
\begin{aligned}
\bar{N}\left(r, \frac{1}{g}\right)+\bar{N}\left(r, \frac{1}{H}\right) & \leq \bar{N}\left(r, \frac{1}{g H}\right)+S(r, f) \\
& =\bar{N}\left(r, \frac{1}{\phi F G}\right)+S(r, f) \\
& \leq \bar{N}\left(r, \frac{1}{\phi}\right)+\bar{N}\left(r, \frac{1}{F}\right)+\bar{N}\left(r, \frac{1}{G}\right)+S(r, f) \\
& \leq T(r, \phi)+\bar{N}\left(r, \frac{1}{F}\right)+\bar{N}\left(r, \frac{1}{G}\right)+S(r, f),
\end{aligned}
$$

from which (2.4.12) follows from substitution of (2.4.22).

Now consider the case where $d \equiv 0$. Then we define $H$ and $\phi$ using (2.1.7) and (2.1.9) by

$$
\begin{gathered}
H=U(F)=V(G), \\
\phi=\frac{g H}{F G}=\frac{P(F) V(G)}{F G}+\frac{Q(G) U(F)}{F G},
\end{gathered}
$$

and $H \not \equiv 0$ by our assumption that $U(F)$ and $V(G)$ are transcendental. Again, $m(r, \phi)=$ $S(r, f)$, but here the only poles of $\phi$ are due to zeros of $F G$, as the poles of $g H$ and $F G$ at poles of $f$ cancel each other out. Thus, (2.4.19) and (2.4.22) hold without the $\bar{N}(r, f)$ term, and on substitution into (2.4.21) yield (2.4.14) and (2.4.15).

QED

\subsubsection{Proof of Theorem 2.2.1}

Assume the hypotheses of the theorem. Suppose first that at least one of $U(F)$ and $V(G)$ is rational. Then by Lemma 2.4.1, either conclusion 2 of the theorem holds, or

$$
\begin{aligned}
T(r, f) & \leq N\left(r, \frac{1}{F}\right)+N\left(r, \frac{1}{G}\right)+S(r, f) \\
& \leq\left(\gamma_{1}+\gamma_{2}\right) T(r, f)+S(r, f)
\end{aligned}
$$

and hence $\gamma_{3}=\gamma_{1}+\gamma_{2} \geq 1$ which implies (2.2.3). We henceforth assume that both $U(F)$ and $V(G)$ are transcendental. Furthermore, if $a-L(c) \equiv b-M(c) \equiv 0$, then we have 
conclusion 1 of the theorem by (2.1.8). We henceforth assume that this is not the case. Then by Lemma 2.4.2,

$$
T(r, f) \leq N\left(r, \frac{1}{g}\right)+\bar{N}(r, f)+\gamma_{0} T(r, f)+S(r, f)
$$

where $\gamma_{0}=\max \left\{\gamma_{1}, \gamma_{2}\right\}$. We now divide the proof into two cases.

\section{$\underline{\text { Case I }}$}

Suppose that $d=U(F)-V(G) \not \equiv 0$ in (2.1.9). Then by Lemma 2.4.3 and (2.2.1), we have

$$
\begin{aligned}
N\left(r, \frac{1}{g}\right)+N\left(r, \frac{1}{H}\right) & \leq \bar{N}(r, f)+2 N\left(r, \frac{1}{F}\right)+2 N\left(r, \frac{1}{G}\right)+S(r, f) \\
& \leq \bar{N}(r, f)+2 \gamma_{3} T(r, f)+S(r, f)
\end{aligned}
$$

where

$$
H=(U(F))^{\prime}-\frac{d^{\prime}}{d} U(F) \not \equiv 0
$$

Now, by (2.1.4) and (2.1.5), $H$ has a representation

$$
H=\left(\left(\frac{d}{d z}-\frac{d^{\prime}}{d}\right)(Y)\right)(f)+\left(\left(\frac{d}{d z}-\frac{d^{\prime}}{d}\right)(U)\right)(a)
$$

as a (possibly non-homogeneous) linear differential polynomial in $f$, of order $k+n+1$, with rational functions as coefficients. Lemmas 2.3.1 and 2.3.2 now give two possibilities, one of which is that $f$ has a representation

$$
f=R\left(y_{1}, \ldots, y_{k+n+2}\right)
$$

where $R$ is a rational function in $k+n+2$ variables and each $y_{j}$ is a (local) solution of

$$
\left(\left(\frac{d}{d z}-\frac{d^{\prime}}{d}\right)(Y)\right)(\omega)=d_{j}\left(\left(\frac{d}{d z}-\frac{d^{\prime}}{d}\right)(U)\right)(a)
$$


where each $d_{j}$ is a constant. By setting $S=Y\left(y_{j}\right)-d_{j} U(a)$, we may rewrite (2.4.28) as

$$
S^{\prime}-\frac{d^{\prime}}{d} S=0
$$

which has solution $S=e_{j} d$ for some constant $e_{j}$. Thus, using (2.1.9),

$$
Y\left(y_{j}\right)=d_{j} U(a)+e_{j} d=\left(d_{j}+e_{j}\right) U(a)-e_{j} V(b)
$$

and so, with $u_{j}, v_{j}, u$ and $v$ as defined, $y_{j}-\left(d_{j}+e_{j}\right) u+e_{j} v$ solves $Y(\omega)=0$ on $\Omega$ and is a linear combination of $u_{1}, \ldots, u_{k}, v_{1}, \ldots, v_{n}$. Thus conclusion 2 of the theorem is satisfied. The other possibility is that

$$
(k+n+1) \bar{N}(r, f) \leq N\left(r, \frac{1}{H}\right)+N(r, f)+S(r, f) .
$$

We combine this with (2.4.27), yielding

$$
\begin{aligned}
N\left(r, \frac{1}{g}\right)+(k+n+1) \bar{N}(r, f) & \leq N\left(r, \frac{1}{g}\right)+N\left(r, \frac{1}{H}\right)+N(r, f)+S(r, f) \\
& \leq \bar{N}(r, f)+\left(2 \gamma_{3}+1\right) T(r, f)+S(r, f),
\end{aligned}
$$

and so

$$
N\left(r, \frac{1}{g}\right)+(k+n) \bar{N}(r, f) \leq\left(2 \gamma_{3}+1\right) T(r, f)+S(r, f),
$$

which leads to

$$
\bar{N}(r, f) \leq \frac{2 \gamma_{3}+1}{k+n} T(r, f)-\frac{1}{k+n} N\left(r, \frac{1}{g}\right)+S(r, f) .
$$

We substitute this in to (2.4.27) and rearrange, ignoring the term $N(r, 1 / H)$, yielding

$$
\frac{k+n+1}{k+n} N\left(r, \frac{1}{g}\right) \leq\left(2 \gamma_{3}+\frac{2 \gamma_{3}+1}{k+n}\right) T(r, f)+S(r, f) .
$$

We then substitute this inequality and (2.4.30) into (2.4.26) to give

$$
T(r, f) \leq\left(\frac{k+n}{k+n+1}\left(2 \gamma_{3}+\frac{2 \gamma_{3}+1}{k+n}\right)+\frac{2 \gamma_{3}+1}{k+n}+\gamma_{0}\right) T(r, f)+S(r, f),
$$

from which (2.2.3) is immediate. 


\section{Case II}

Now suppose that $d \equiv 0$. Then by Lemma 2.4 .3 we have

$$
\begin{aligned}
N\left(r, \frac{1}{g}\right)+N\left(r, \frac{1}{H}\right) & \leq 2 N\left(r, \frac{1}{F}\right)+2 N\left(r, \frac{1}{G}\right)+S(r, f) \\
& \leq 2 \gamma_{3} T(r, f)+S(r, f),
\end{aligned}
$$

where $H=U(F)$. Now, by (2.1.4) and (2.1.5), $H$ has a representation

$$
H=Y(f)+U(a)
$$

as a (possibly non-homogeneous) linear differential polynomial in $f$, of order $k+n$, with rational functions as coefficients. Lemmas 2.3.1 and 2.3.2 now give two possibilities, one of which is that $f$ has a representation

$$
f=R\left(y_{1}, \ldots, y_{k+n+1}\right)
$$

where $R$ is a rational function in $k+n+1$ variables and each $y_{j}$ is a (local) solution of

$$
Y(\omega)=d_{j} U(a)
$$

where each $d_{j}$ is a constant, so that $y_{j}-d_{j} u$ solves $Y(\omega)=0$, and thus conclusion 2 of the theorem is satisfied. The other possibility is that

$$
(k+n) \bar{N}(r, f) \leq N\left(r, \frac{1}{H}\right)+N(r, f)+S(r, f) .
$$

Substituting in (2.4.31), we obtain

$$
\bar{N}(r, f) \leq \frac{2 \gamma_{3}+1}{k+n} T(r, f)+S(r, f) .
$$

We now substitute (2.4.31) into (2.4.26) to give

$$
\left(1-2 \gamma_{3}-\gamma_{0}\right) T(r, f) \leq \bar{N}(r, f)+S(r, f) .
$$


We substitute this into (2.4.32) and thus obtain

$$
\left(1-2 \gamma_{3}-\gamma_{0}\right) T(r, f) \leq \frac{2 \gamma_{3}+1}{k+n} T(r, f)+S(r, f),
$$

which is a stronger condition than (2.2.3).

QED

\subsubsection{Proof of Theorem 2.2.2}

Assume the hypotheses of the theorem. If either $U(F)$ or $V(G)$ is rational, then by Lemma 2.4.1, either conclusion 2 of Theorem 2.2.2 holds, or by (2.2.4) and (2.4.2) we have $T(r, f)=S(r, f)$, which is a contradiction. Henceforth assume that both $U(F)$ and $V(G)$ are transcendental. Then if $a-L(c) \equiv b-M(c) \equiv 0$ then, by (2.1.8), Conclusion 1 of Theorem 2.2.2 holds. Assume henceforth without loss of generality that $a-L(c) \not \equiv 0$. Then by Lemmas 2.4.2 and 2.4.3 and (2.2.4),

$$
\begin{aligned}
T(r, f) & \leq(n+1) \bar{N}\left(r, \frac{1}{g}\right)+\bar{N}(r, f)+S(r, f) \\
& \leq(n+2) \bar{N}(r, f)+S(r, f),
\end{aligned}
$$

and in particular,

$$
\bar{N}(r, f) \neq S(r, f)
$$

We define $\lambda_{j}=\psi^{(j)} / \psi$ for $j \geq 0$, and in particular, $\lambda=\lambda_{1}=\psi^{\prime} / \psi$, where

$$
\psi=\frac{L(f)+a}{M(f)+b}=\frac{F}{G}
$$

If $\psi \equiv c_{1}$ for some constant $c_{1}$, and thus $\lambda \equiv 0$, then

$$
L(f)+a=F=c_{1} G=c_{1}(M(f)+b) .
$$

Since the equations $L(\omega)=0$ and $M(\omega)=0$ have no non-trivial solutions in common, $f$ solves a (possibly non-homogeneous) linear differential equation with rational coefficients. 
Thus, since all terms in the differential equation have differing weight, poles cannot cancel out except at the finitely many poles of the coefficients, and thus $f$ has only finitely many poles, contradicting (2.4.33). Thus $\lambda \not \equiv 0$. Since $F$ and $G$ have the same leading term, all but finitely many poles of $f$ are 1-points of $\psi$ by (2.1.3) and (2.1.5). Poles of $\lambda$ can come from zeros of $\psi$ (which generates a zero of $\psi^{\prime}$ of multiplicity one less and thus a simple pole of $\lambda$ ) or poles of $\psi^{\prime}$ (which are also poles of $\psi$ of multiplicity one less, and thus a simple pole of $\lambda$ ), hence we have that

$$
N(r, \lambda)=\bar{N}\left(r, \frac{1}{\psi}\right)+\bar{N}(r, \psi)
$$

and so by (2.2.4),

$$
\begin{aligned}
N(r, \lambda) & \leq \bar{N}\left(r, \frac{1}{F}\right)+\bar{N}\left(r, \frac{1}{G}\right)+O(\log r) \\
& =S(r, f) .
\end{aligned}
$$

Since $\lambda$ is the quotient of a function and its derivative, we have that $m(r, \lambda)=S(r, \psi)$ by the Lemma of the Logarithmic Derivative (1.3.2). Furthermore, by Lemmas 1.2.2 and 1.3.6, we have that $T(r, \psi)=O(T(r, f))$, and so any term which is $S(r, \psi)$ is also $S(r, f)$. Thus,

$$
T(r, \lambda)=S(r, f)
$$

Now, for $j \in \mathbb{N}$,

$$
\begin{aligned}
F^{(j)}=(\psi G)^{(j)} & =\sum_{\phi=0}^{j}\left(\begin{array}{l}
j \\
\phi
\end{array}\right) \psi^{(j-\phi)} G^{(\phi)} \\
& =\psi \sum_{\phi=0}^{j}\left(\begin{array}{l}
j \\
\phi
\end{array}\right) \lambda_{j-\phi} G^{(\phi)} .
\end{aligned}
$$

Let $U$ and $V$ be as in (2.1.4), and write

$$
U(F)=\sum_{j=0}^{n} p_{j} F^{(j)}, \quad V(G)=\sum_{j=0}^{n} q_{j} G^{(j)}
$$


with $p_{n}=q_{n}=1$. Then $(2.1 .9)$ gives

$$
\begin{aligned}
V(G)+d=U(F)=\sum_{j=0}^{n} p_{j} F^{(j)} & =\sum_{j=0}^{n} p_{j} \psi \sum_{t=0}^{j}\left(\begin{array}{l}
j \\
t
\end{array}\right) \lambda_{j-t} G^{(t)} \\
& =\psi \sum_{j=0}^{n} p_{j} \sum_{t=0}^{n}\left(\begin{array}{l}
j \\
t
\end{array}\right) \lambda_{j-t} G^{(t)} \\
& =\psi \sum_{t=0}^{n} \sum_{j=0}^{n} p_{j}\left(\begin{array}{l}
j \\
t
\end{array}\right) \lambda_{j-t} G^{(t)} \\
& =\psi \sum_{t=0}^{n} \sum_{j=t}^{n} p_{j}\left(\begin{array}{l}
j \\
t
\end{array}\right) \lambda_{j-t} G^{(t)}
\end{aligned}
$$

using the property that $\left(\begin{array}{l}\mu \\ \nu\end{array}\right)=0$ for $\mu \notin[0, \nu]$. Now, suppose that $z_{0}$ is large and a pole of $f$ of order $m$. Then $z_{0}$ is a pole of $G$ of order $m+n$, and for $0 \leq l \leq n, X_{l}=G^{(l)} / G^{(n)}$ has a zero of order $n-l$ at $z_{0}$. Further, we have $\psi\left(z_{0}\right)=1$ and $\lambda_{l}\left(z_{0}\right) \in \mathbb{C} \forall l \geq 0$. Thus at $z_{0}$, by the definition of $V(G)$,

$$
\frac{V(G)+d}{G^{(n)}}=1+q_{n-1} X_{n-1}+M_{1}
$$

where $M_{j}$ will denote functions with a zero of multiplicity at least two at $z_{0}$. But we also have, using (2.1.9) and (2.4.40),

$$
\frac{V(G)+d}{G^{(n)}}=\frac{U(F)}{G^{(n)}}=\psi\left(1+\left(p_{n-1}+n \lambda\right) X_{n-1}+M_{2}\right) .
$$

Now, $G$ has a pole of multiplicity $m+n$ at $z_{0}$, and so $G^{(j)}$ has a pole of multiplicity $m+n+j$, and we may write, as $z \rightarrow z_{0}$,

$$
X_{n-1} \sim \frac{\left(z-z_{0}\right)^{-(m+2 n-1)}}{-(m+2 n-1)\left(z-z_{0}\right)^{-(m+2 n)}}=\frac{-\left(z-z_{0}\right)}{m+2 n-1} .
$$

We then differentiate, giving $X_{n-1}^{\prime}\left(z_{0}\right)=-(m+2 n-1)^{-1}$. We have using $(2.4 .41)$ and (2.4.42),

$$
1+q_{n-1} X_{n-1}+M_{1}=\psi\left(1+\left(p_{n-1}+n \lambda\right) X_{n-1}+M_{2}\right),
$$

which we now differentiate to give, setting $\widetilde{p}=p_{n-1}+n \lambda$ and using $\lambda=\psi^{\prime} / \psi$,

$$
q_{n-1}^{\prime} X_{n-1}+q_{n-1} X_{n-1}^{\prime}+M_{1}^{\prime}=\lambda \psi\left(1+\widetilde{p} X_{n-1}+M_{2}\right)+\psi\left(\widetilde{p} X_{n-1}^{\prime}+\widetilde{p} X_{n-1}+M_{2}^{\prime}\right) .
$$


We now substitute in $\psi\left(z_{0}\right)=1, X_{n-1}\left(z_{0}\right)=0, X_{n-1}^{\prime}\left(z_{0}\right)=-(m+2 n-1)^{-1}$ and $M_{j}\left(z_{0}\right)=M_{j}^{\prime}\left(z_{0}\right)=0$, then rearrange to give, at $z_{0}$,

$$
\begin{aligned}
\lambda-\frac{n \lambda}{m+2 n-1} & =\frac{p_{n-1}-q_{n-1}}{m+2 n-1} \\
\lambda & =\frac{p_{n-1}-q_{n-1}}{m+n-1} .
\end{aligned}
$$

Now, suppose there exists no $m \in \mathbb{N}$ such that $\lambda \equiv \frac{p_{n-1}-q_{n-1}}{m+n-1}$. We define $N^{m}(r, f)$ to be the counting function $N(r, f)$ restricted to those poles of multiplicity $m$. We rearrange (2.4.47) to give

$$
\Lambda=\lambda-\frac{p_{n-1}-q_{n-1}}{m+n-1}=0
$$

and so

$$
\bar{N}^{m}(r, f) \leq N\left(r, \frac{1}{\Lambda}\right)+S(r, f)
$$

where the $S(r, f)$ term takes account of the finitely many poles excluded from our analysis above. But

$$
T(r, \Lambda) \leq T(r, \lambda)+T\left(r, p_{n-1}\right)+T\left(r, q_{n-1}\right)+S(r, f),
$$

which, by (2.4.37) and the fact that the $p_{j}$ and $q_{j}$ are rational functions, is itself $S(r, f)$. Thus, since $\Lambda \not \equiv 0$ by assumption,

$$
\bar{N}^{m}(r, f) \leq T(r, \Lambda)+S(r, f)=S(r, f) .
$$

Now, let $\varepsilon>0$. For each $\kappa \in \mathbb{N}$ we may choose $r_{\kappa}$ such that

$$
\sum_{m=1}^{\kappa} \bar{N}^{m}(r, f)=S(r, f)=o(T(r, f)) \leq \frac{1}{\kappa} T(r, f)
$$

for $r \geq r_{\kappa}$ outside some set $E_{\kappa}$ of measure at most $\kappa^{-2}$. We further assume that $r_{\kappa+1} \geq r_{\kappa}$ $\forall \kappa \in \mathbb{N}$. Then $E=\bigcup_{\kappa \in \mathbb{N}} E_{\kappa}$ has finite measure. Let $\kappa$ be big enough that $2 \kappa^{-1} \leq \varepsilon$, 
and $r$ large and not in $E$. Then $r \geq r_{\kappa}, r \notin E_{\kappa}$, so

$$
\begin{aligned}
\sum_{m \leq \kappa} \bar{N}^{m}(r, f) & \leq \frac{1}{\kappa} T(r, f) \leq \frac{\varepsilon}{2} T(r, f) \\
\sum_{m>\kappa} \bar{N}^{m}(r, f) \leq \frac{1}{\kappa} N(r, f) & \leq \frac{1}{\kappa} T(r, f) \leq \frac{\varepsilon}{2} T(r, f),
\end{aligned}
$$

and so

$$
\bar{N}(r, f) \leq \varepsilon T(r, f) .
$$

This holds for all sufficiently large $r \notin E$, and so

$$
\bar{N}(r, f)=S(r, f),
$$

which contradicts (2.4.33).

It follows that there is some $m \in \mathbb{N}$ such that

$$
\lambda \equiv \frac{p_{n-1}-q_{n-1}}{m+n-1},
$$

and so $\lambda$ is a rational function. But, since $\psi^{\prime}=\lambda \psi$, we have by Lemma 1.4.3 that

$$
\psi=\frac{F}{G}=R e^{P}
$$

for some rational function $R$ and polynomial $P$, and thus $\psi$ has only finitely many poles and zeros. Suppose that $F$ has infinitely many zeros of multiplicity greater than $n$. Then all but finitely many of these are zeros of $G$ of the same multiplicity, since $\psi$ has finitely many zeros and poles, and they are zeros of $U(F)$ and $V(G)$, and thus of $d$ by (2.1.9). Thus, there are infinitely many zeros of $d$, and so, since $d$ is rational, $d \equiv 0$.

We now follow a slight variation on our previous argument. Suppose $z_{0}$ is large and a zero of $G$ of multiplicity $\mu \geq n$. Then $X_{j}=G^{(j)} / G^{(n)}$ has a zero of multiplicity $n-j$ at $z_{0}$ for $0 \leq j \leq n$. As before, $X_{n-1}\left(z_{0}\right)=0$, but now, as $z \rightarrow z_{0}$,

$$
X_{n-1}(z) \sim \frac{\left(z-z_{0}\right)^{\mu-n+1}}{(\mu-n+1)\left(z-z_{0}\right)^{\mu-n}}
$$


and so $X_{n-1}^{\prime}\left(z_{0}\right)=(\mu-n+1)^{-1}$. We again have (2.4.41) and (2.4.42), and hence (2.4.44) and (2.4.45). We also have that $\psi\left(z_{0}\right)=1$ by (2.4.41) and (2.4.42). We substitute this and (2.4.59) into (2.4.45), giving

$$
\frac{q_{n-1}}{\mu-n+1}=\lambda+\frac{p_{n-1}+n \lambda}{\mu-n+1}
$$

which we rearrange to find

$$
\lambda=\frac{q_{n-1}-p_{n-1}}{\mu+1}
$$

However, we already have an identity for $\lambda$ by (2.4.57), which we equate to give

$$
\begin{aligned}
\frac{q_{n-1}-p_{n-1}}{\mu+1}=\lambda & \equiv \frac{p_{n-1}-q_{n-1}}{m+n-1} \\
\frac{-1}{\mu+1} & =\frac{1}{m+n-1} .
\end{aligned}
$$

But, $\mu>0$ and $m+n>1$, and so we are trying to equate one number which is strictly positive and another which is strictly negative - clearly impossible. Thus, our supposition that there are infinitely many such zeros of multiplicity greater than $n$ false, and so there are only finitely many of these zeros, which thus contribute $O(\log r)=S(r, f)$ to $N(r, 1 / F)$ and $N(r, 1 / G)$. Hence, by $(2.2 .4)$,

$$
\begin{aligned}
& N\left(r, \frac{1}{F}\right) \leq n \bar{N}\left(r, \frac{1}{F}\right)+S(r, f)=S(r, f), \\
& N\left(r, \frac{1}{G}\right) \leq n \bar{N}\left(r, \frac{1}{G}\right)+S(r, f)=S(r, f),
\end{aligned}
$$

and thus we can apply Langley's Proposition 2.1.4 with $k=n$ and the conclusions of Theorem 2.2.2 follow immediately.

\subsubsection{Proof of Theorem 2.2.3}

We begin by stating a refinement of Lemma 2.3.1 from [23]. 


\section{Lemma 2.4.4 $[23]$}

Let $0<\varepsilon<1$, and let $L_{0}$ be a homogeneous linear differential operator of order $p \geq 2$ with rational functions for coefficients. Let $h$ be transcendental and meromorphic in the plane, and $H=L_{0}(h)$. Then at least one of the following two conclusions holds:

1. $h$ is rational in $p$ (local) solutions of $L_{0}(\omega)=0$;

2. the functions $h$ and $H$ satisfy

$$
N(r, H) \leq C \bar{N}\left(r, \frac{1}{H}\right)+(2+\varepsilon) N(r, h)+S(r, f)
$$

where

$$
C \leq(2+\varepsilon) \exp \frac{4(p-1)}{\log (1+\varepsilon)}
$$

We note here that $H$ is required to be a homogeneous differential polynomial in $h$. We may now begin the proof of Theorem 2.2.3.

Assume the hypotheses of Theorem 2.2.3. By Lemma 2.4.1, if either $U(F)$ or $V(G)$ are rational then either conclusion 2 of our theorem holds, or (2.4.2) holds, which, by (2.2.4), means that $T(r, f) \leq S(r, f)$, which is a contradiction. Assume henceforth that $U(F)$ and $V(G)$ are transcendental. By Lemma 2.4.3 and (2.2.4),

$$
\bar{N}\left(r, \frac{1}{g}\right) \leq \bar{N}(r, f)+S(r, f) .
$$

We combine this with (2.4.5) of Lemma 2.4.2, which holds under our assumption that $a-L(c) \not \equiv 0$, to yield

$$
N(r, f) \leq T(r, f) \leq(k+2) \bar{N}(r, f)+S(r, f) .
$$

We apply Lemma 2.4.4 with $p=n, h=f, H=G=M(f)$ and $\varepsilon$ small. If conclusion 1 of the lemma holds, then $f$ is a rational function in solutions of $M(\omega)=0$, and so 
conclusion 2 of the theorem holds. Assume this is not the case. Then (2.4.66) holds, and we apply (2.2.4), and thus obtain

$$
N(r, G) \leq(2+\varepsilon) N(r, f)+S(r, f)
$$

Now, the poles of $G$ are caused either by poles of the coefficients, which since they are rational contribute $S(r, f)$, or by poles of $f$, where if $z_{0}$ is large and a pole of $f$ of order $m$, then it is a pole of $G$ of order $m+n$. Thus,

$$
N(r, G)=N(r, f)+n \bar{N}(r, f)+S(r, f)
$$

which we substitute into (2.4.68), giving

$$
n \bar{N}(r, f) \leq(1+\varepsilon) N(r, f)+S(r, f)
$$

and hence by substitution of (2.4.67),

$$
n \bar{N}(r, f) \leq(1+\varepsilon)(k+2) \bar{N}(r, f)+S(r, f)
$$

By (2.4.67) we have $\bar{N}(r, f) \neq S(r, f)$, and so $n \leq(1+\varepsilon)(k+2)$. Since $\varepsilon$ may be chosen arbitrarily small, this contradicts (2.2.5).

QED

\subsubsection{Proof of Theorem 2.2.4}

We present the proof for $a-L(c) \not \equiv 0$; the proof when $b-M(c) \not \equiv 0$ follows along the same lines.

Suppose that one of $U(F)$ or $V(G)$ is rational. Then, as noted in the proof of Lemma 2.4.1, $f$ has finitely many poles. Now, suppose that $U(F)$ and $V(G)$ are transcendental. Then by (2.4.4) and Lemma 2.4.3,

$$
T(r, f) \leq 2 \bar{N}(r, f)+2 N\left(r, \frac{1}{F}\right)+2 N\left(r, \frac{1}{G}\right)+\bar{N}\left(r, \frac{1}{F}\right)+S(r, f),
$$


which, by (2.1.10), reduces to

$$
T(r, f) \leq 2 \bar{N}(r, f)+S(r, f)
$$

and in particular $\bar{N}(r, f) \neq S(r, f)$. Applying Lemma 2.3.2 with $p=n, Q_{p}=M$ and $H=G$ gives either conclusion 3 of the theorem, or, by substitution of (2.4.69) and (2.1.10),

$$
\begin{aligned}
n \bar{N}(r, f) & \leq N\left(r, \frac{1}{G}\right)+N(r, f)+S(r, f) \\
& \leq 2 \bar{N}(r, f)+S(r, f),
\end{aligned}
$$

from which conclusion 2 is immediate. 


\section{Chapter 3}

\section{Non-linear homogeneous}

\section{differential polynomials in $f$ and}

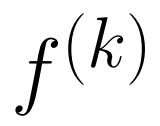

In this chapter, we apply lemmas of Mues and Steinmetz from [24] to non-linear homogeneous differential polynomials in the meromorphic function $f$ and $f^{(k)}$ with coefficients which are $O(\log r)+o(T(r, f))$ in order to find sufficient conditions for $f$ to be of the form $R e^{P}$ where $R$ is a rational function and $P$ is a polynomial. This work was published in Computational Methods and Function Theory [6].

\subsection{Introduction and results}

We consider non-linear homogeneous differential polynomials $F$ in a meromorphic function $f$ and $f^{(k)}$ with restrictions on the frequency of the zeros, and from there attempt to determine the form of $f$. Other results on homogeneous differential polynomials have been proved by various authors, for instance in [1], [31] and [33] (see also Chapter 4). 
We write $\lambda(r, h)$ for any term which is $O(\log r)+o(T(r, h))$ nearly everywhere (n.e.), that is, outside some set of finite measure.

Let $f$ be a transcendental meromorphic function in the plane. We define

$$
u=\frac{f}{f^{(k)}}
$$

for some $k \geq 1$. Further, let

$$
F=f^{n}+\sum_{j=0}^{n-2} c_{j} f^{j}\left(f^{(k)}\right)^{n-j}
$$

be a homogeneous non-linear differential polynomial in $f$ and $f^{(k)}$, with coefficients $c_{j}$ such that $T\left(r, c_{j}\right)=\lambda(r, u)$. We may further rewrite (3.1.2) as

$$
F=\left(f^{(k)}\right)^{n} \psi
$$

where

$$
\psi=u^{n}+\sum_{j=0}^{n-2} c_{j} u^{j} .
$$

We will assume in all results that $\bar{N}(r, 1 / \psi)=\lambda(r, u)$.

Our first result is obtained by placing a restriction on the frequency of the distinct zeros of $f$.

\section{Theorem 3.1.1}

Let $u$ be as in (3.1.1) with $k \geq 2$, and let $\psi$ be as in (3.1.3). Suppose that $\bar{N}(r, 1 / f)+\bar{N}(r, 1 / \psi)=\lambda(r, u)$, and that there is at least one $j$ such that $c_{j} \not \equiv 0$. Then $f=R e^{P}$, where $R$ is a rational function and $P$ a polynomial. 
Our second result is obtained by placing a restriction on the frequency of the zeros of $f^{(k)}$.

\section{Theorem 3.1.2}

Let $u$ be as in (3.1.1) with $k \geq 1$, and let $\psi$ be as in (3.1.3). Suppose that $\bar{N}\left(r, 1 / f^{(k)}\right)+\bar{N}(r, 1 / \psi)=\lambda(r, u)$, and that there is at least one $j$ such that $c_{j} \not \equiv 0$. Then $f=R e^{P}$, where $R$ is a rational function and $P$ a polynomial.

Our third theorem drops the restriction on the zeros of $f$ and $f^{(k)}$, instead replacing it with a requirement on the Nevanlinna deficiency $\delta(\alpha, f)$ to give a much stronger conclusion.

\section{Theorem 3.1.3}

Let $u$ be as in (3.1.1) with $k \geq 1$, and let $\psi$ be as in (3.1.3). Suppose that $\alpha \in \mathbb{C} \backslash\{0\}$ is such that $\delta(\alpha, f)>0$, that $\bar{N}(r, 1 / \psi)=\lambda(r, u)$, and that there is at least one $j$ such that $c_{j} \not \equiv 0$. Then $f$ is a rational function.

\subsection{Lemmas}

We begin by stating some useful lemmas, assuming throughout this section that $\psi$ is as in (3.1.3), that $\bar{N}(r, 1 / \psi)=\lambda(r, u)$, and that there is no constant $c$ such that $\psi \equiv c u^{n}$. This requirement is slightly stronger than assuming some $c_{j} \not \equiv 0$. We first state a slightly modified lemma from [10], which provides an important step in our working. 


\section{Lemma 3.2.1 - Clunie's Lemma $[10]$}

Suppose that $h^{n} P[h]=Q[h]$, where $h$ is meromorphic in the plane and $P[h]$ and $Q[h]$ are polynomials in $h$ and its derivatives with meromorphic functions $c$ satisfying $T(r, c)=\lambda(r, h)$ as coefficients, $Q[h]$ being of degree $n$ at most. Then

$$
m(r, P[h])=\lambda(r, h)
$$

Remark: Clunie proved this as

$$
m(r, P[h])=O(\log r+\log T(r, h)+\mathcal{T}(r))
$$

where $\mathcal{T}(r)$ is the maximum of the characteristics of the coefficients in $P[h]$ and $Q[h]$. Given our restriction on $T(r, c)$, and since $\log T(r, h)=o(T(r, h))$, it is clear that this is $\lambda(r, h)$.

We now move on to a lemma from [24], which provides the main thrust of our argument by estimating the Nevanlinna functionals of $u$ and $1 / u$.

\section{Lemma 3.2.2 [24]}

With the assumptions of this section on $\psi$ and $u$, we have

$$
\begin{array}{r}
m(r, u)=\lambda(r, u), \\
m(r, 1 / u)=\lambda(r, u), \\
N_{1}(r, u)=\lambda(r, u), \\
N_{1}(r, 1 / u)=\lambda(r, u),
\end{array}
$$

where $N_{1}(r, u)=N(r, u)-\bar{N}(r, u)$, and thus may be considered to only count multiple poles of $u$. 
Remark: It is important to note that the definition of $N_{1}(r, f)$ used here is not the same as in the statement of the Second Fundamental Theorem (1.3.4). The notation $N_{1}(r, f)$ was used in [24] and so we retain it for simplicity. Mues and Steinmetz proved that the above holds with $\lambda(r, u)$ replaced by $S(r, u)$. We include a full proof below, but note that if $u$ is transcendental, then $\lambda(r, u)=S(r, u)$, and so we may simply apply the original result. If however $u$ is rational, then $T(r, u)=O(\log r)=\lambda(r, u)$, and so the result is trivial.

Proof:

Let

$$
a=u^{\prime}-\frac{1}{n} \frac{\psi^{\prime}}{\psi} u
$$

We will show that

$$
T(r, a)=\lambda(r, u)
$$

Since $\psi \not \equiv u^{n}$, we have by $(3.1 .3)$

$$
\psi=u^{n}+R[u]
$$

where $R[u] \not \equiv 0$ is a polynomial in $u$ with coefficients satisfying $T\left(r, c_{j}\right)=\lambda(r, u)$. Differentiating (3.2.7), we have

$$
\psi^{\prime}=n u^{n-1} u^{\prime}+R[u]^{\prime},
$$

which we may rewrite as

$$
u^{n-1} P[u]=Q[u]
$$

where $P$ and $Q$ are given by

$$
P[u]=n u^{\prime}-\frac{\psi^{\prime}}{\psi} u \quad \text { and } \quad Q[u]=\frac{\psi^{\prime}}{\psi} R[u]-R[u]^{\prime}
$$


and the degree of $Q$ is at most $n-2$ in $u$ and its derivatives. We define

$$
a=u^{\prime}-\frac{1}{n} \frac{\psi^{\prime}}{\psi} u=\frac{P[u]}{n}
$$

and apply Clunie's Lemma to (3.2.8). This gives us that

$$
m(r, P[u])=m(r, n a)=m(r, a)+O(1)=\lambda(r, u) .
$$

Suppose that $a \equiv 0$. Then by (3.2.9) we have that $\psi=c u^{n}$ for some constant $c$, which contradicts our assumption that no such $c$ exists. Hence $a \not \equiv 0$.

Now, let $z_{0}$ be a pole of $a$ of multiplicity $\mu$. If $u\left(z_{0}\right) \neq \infty$, then $\mu=1$ and either $\psi\left(z_{0}\right)=0$, or $\psi\left(z_{0}\right)=\infty$ and thus there must be some $j$ such that $c_{j}$ has a pole at $z_{0}$. If however $u$ has a pole at $z_{0}$ of multiplicity $\nu$, we may assume the $c_{j}$ have poles of order at most $\eta$, and by (3.2.8),

$$
(n-1) \nu+\mu \leq 1+(n-2) \nu+\eta, \quad \text { and so } \quad \mu \leq 1-\nu+\eta \leq \eta,
$$

where the terms in the first inequality come from $u^{n-1}, a, \psi^{\prime} / \psi$, the powers of $u$ in $R[u]$, and the $c_{j}$ respectively. Hence,

$$
N(r, a) \leq \bar{N}\left(r, \frac{1}{\psi}\right)+\sum_{j=0}^{n-2} N\left(r, c_{j}\right) \leq \lambda(r, u)
$$

by our hypothesis on the zeros of $\psi$ and the characteristics of the coefficients $c_{j}$. Thus, combining this with (3.2.10), we return (3.2.6) and so prove the existence of this function.

Taking (3.2.8), we divide through by $n a=P[u]$, yielding $u^{n-2} u=Q[u] / n a$, to which we apply Clunie's Lemma, and so (3.2.2) follows.

Now, divide (3.2.9) by au, hence

$$
\begin{aligned}
m\left(r, \frac{1}{u}\right) & =m\left(r, \frac{1}{a}\left(\frac{u^{\prime}}{u}-\frac{1}{n} \frac{\psi^{\prime}}{\psi}\right)\right) \\
& \leq T(r, a)+m\left(r, \frac{u^{\prime}}{u}\right)+m\left(r, \frac{\psi^{\prime}}{\psi}\right)+O(1)
\end{aligned}
$$


But, by the Lemma of the Logarithmic Derivative (1.3.2),

$$
m\left(r, u^{\prime} / u\right)=O\left(\log r+\log ^{+} T(r, u)\right)=\lambda(r, u)
$$

outside a set of finite measure. Further, since $T(r, \psi)=O\left(T(r, u)+\sum T\left(r, c_{j}\right)\right)$, we have $m\left(r, \psi^{\prime} / \psi\right)=\lambda(r, u)$. Thus, by (3.2.6), (3.2.3) follows.

Let $z_{0}$ be a pole of $u$ of order $\nu \geq 2$, and suppose the $c_{j}$ have poles at $z_{0}$ of order at most $\eta$, and that at $z_{0}, a$ has a pole of order $\mu>0$ or has a zero of order $-\mu \geq 0$. Thus, (3.2.8) gives (3.2.11) again, and we have that $\nu-1 \leq \eta-\mu \leq \eta+\max \{0,-\mu\}$, and so by $(3.2 .6)$

$$
\begin{aligned}
N_{1}(r, u) & \leq N\left(r, \frac{1}{a}\right)+\sum_{j=0}^{n-2} N\left(r, c_{j}\right) \\
& \leq T(r, a)+\sum_{j=0}^{n-2} T\left(r, c_{j}\right) \\
& =\lambda(r, u),
\end{aligned}
$$

thus proving (3.2.4).

Finally, suppose $z_{0}$ is a zero of $u$ of order $\nu \geq 2$. Then $a$ has a zero of multiplicity at least $\nu-1$ at $z_{0}$, and so by (3.2.6)

$$
N_{1}(r, u) \leq N\left(r, \frac{1}{a}\right) \leq T(r, a)+O(1)=\lambda(r, u)
$$

QED

\section{Lemma 3.2.3}

For any meromorphic function $h$, we have

$$
N^{2+}(r, h) \leq 2 N_{1}(r, h)
$$

where $N^{2+}(r, h)$ counts only multiple poles of $h$, each according to multiplicity. 
Proof:

If $z_{0}$ is a pole of $h$ of multiplicity $j>0$, it adds $2(j-1)$ to $2 n_{1}(r, h)$. Since $2(j-1) \geq j$ for all $j \geq 2$, we get $n^{2+}(r, h) \leq 2 n_{1}(r, h)$, and thus $N^{2+}(r, h) \leq 2 N_{1}(r, h)$.

QED

Our next lemma extends Lemma 1.4.3 to the quotient $f / f^{(k)}$, subject to conditions on the frequency of zeros of either numerator or denominator.

\section{Lemma 3.2.4}

If $u=f / f^{(k)}$ is a rational function for some $k \geq 1$ and either $f$ or $f^{(k)}$ has finitely many zeros, then $f=R e^{P}$, where $R$ is a rational function and $P$ is a polynomial.

Remark: This does not hold without the restriction on the number of zeros, as $f=\cos z$ solves $f^{\prime \prime}=-f$ yet has infinitely many zeros.

Proof:

By Lemma 1.4.3, it is sufficient to prove that $v=f^{\prime} / f$ is a rational function. A pole of $f$ gives rise to a zero of $u$, and hence $f$ has only finitely many poles since $u$ is rational. Further, since at least one of $f$ and $f^{(k)}$ has finitely many zeros, this is true of both, because $u$ has finitely many zeros and poles. We aim to show that $v=f^{\prime} / f$ is rational, from whence Lemma 1.4.3 proves the result. We have that

$$
\begin{aligned}
N(r, v)=N\left(r, \frac{f^{\prime}}{f}\right) & =\bar{N}(r, f)+\bar{N}\left(r, \frac{1}{f}\right) \\
& =O(\log r) .
\end{aligned}
$$

Using Lemma 3.5 from [16], we may write

$$
\frac{1}{u}=v^{k}+S[v]
$$


where $S$ is a differential polynomial in $v$ with constant coefficients, of degree at most $k-1$. We rewrite this as

$$
v^{k-1} v=\frac{1}{u}-S[v],
$$

and since $u$ is rational we have $T(r, u)=\lambda(r, v)$. Thus, Clunie's Lemma implies

$$
m(r, v)=\lambda(r, v)
$$

and so, using (3.2.13),

$$
T(r, v)=\lambda(r, v)=O(\log r)+o(T(r, v)),
$$

and hence $v$ is rational.

QED

\section{Lemma 3.2.5}

Suppose that $h$ is meromorphic in the plane and that

$$
h^{m}+d_{m-1} h^{m-1}+\ldots+d_{1} h+d_{0} \equiv 0
$$

where the coefficients $d_{j}$ are meromorphic functions such that $T\left(r, d_{j}\right)=\lambda(r, h)$. Then $h$ is a rational function.

We omit the proof of this lemma as it is well-known and quite elementary. We now prove one final lemma concerning the Nevanlinna deficiency $\delta(\alpha, f)$.

\section{Lemma 3.2.6}

Suppose that the transcendental meromorphic function $f$ has a value $\alpha \in \mathbb{C} \backslash\{0\}$ such that

$$
\delta=\delta(\alpha, f)=1-\limsup _{r \rightarrow \infty} \frac{N(r, 1 /(f-\alpha))}{T(r, f)}>0 .
$$


Then

$$
T(r, f)+T(r, u)=O(m(r, u)) \quad \text { (n.e.). }
$$

Proof:

We rewrite

$$
\frac{1}{f-\alpha}=\frac{f}{f^{(k)}} \frac{f^{(k)}}{f(f-\alpha)}=\frac{f}{\alpha f^{(k)}}\left(\frac{f^{(k)}}{f-\alpha}-\frac{f^{(k)}}{f}\right) .
$$

By the First Fundamental Theorem (1.3.1), $T(r, f)=T(r, 1 /(f-\alpha))+O(1)$, and so by (3.2.15) and the Lemma of the Logarithmic Derivative (1.3.2),

$$
\begin{aligned}
(\delta-o(1)) T(r, f) & \leq m\left(r, \frac{1}{f-\alpha}\right) \\
& \leq m\left(r, \frac{f}{f^{(k)}}\right)+m\left(r, \frac{f^{(k)}}{f-\alpha}\right)+m\left(r, \frac{f^{(k)}}{f}\right)+O(1) \\
& =m\left(r, \frac{f}{f^{(k)}}\right)+o(T(r, f)) \quad \text { (n.e.) }
\end{aligned}
$$

and so, outside a set of finite measure, $m(r, u)=m\left(r, f / f^{(k)}\right) \geq(\delta-o(1)) T(r, f)$.

However, we also note that

$$
T(r, u)=T\left(r, \frac{f}{f^{(k)}}\right) \leq T(r, f)+T\left(r, f^{(k)}\right)=O(T(r, f)) \quad \text { (n.e.) }
$$

and hence

$$
(\delta-o(1)) T(r, f) \leq m(r, u) \leq T(r, u) \leq O(T(r, f)) \quad \text { (n.e.) }
$$

from which (3.2.16) follows. 


\subsection{Proof of the theorems}

Proof of Theorem 3.1.1:

Suppose that $u$ is rational. Then $\lambda(r, u)=O(\log r)$, and thus $f$ has only finitely many zeros. Hence by Lemma $3.2 .4, f=R e^{P}$.

Now suppose that $u$ is transcendental. Then there exists no $c \in \mathbb{C}$ such that $\psi \equiv c u^{n}$, since otherwise we have an identity of the form (3.2.14), and produce a contradiction via Lemma 3.2.5. Using the First Fundamental Theorem of Nevanlinna Theory (1.3.1),

$$
\begin{aligned}
T(r, u) & =T\left(r, \frac{1}{u}\right)+O(1) \\
& =N\left(r, \frac{1}{u}\right)+m\left(r, \frac{1}{u}\right)+O(1) \\
& =N^{1}\left(r, \frac{1}{u}\right)+N^{2+}\left(r, \frac{1}{u}\right)+m\left(r, \frac{1}{u}\right)+O(1),
\end{aligned}
$$

where $N^{1}(r, 1 / u)$ counts only simple zeros of $u$. By (3.2.3), (3.2.5) and Lemma 3.2.3, we have

$$
N^{2+}\left(r, \frac{1}{u}\right)+m\left(r, \frac{1}{u}\right) \leq 2 N_{1}\left(r, \frac{1}{u}\right)+\lambda(r, u) \leq \lambda(r, u)
$$

Since for $u$ to have a simple zero, $f$ must have a zero,

$$
N^{1}\left(r, \frac{1}{u}\right) \leq \bar{N}\left(r, \frac{1}{f}\right)=\lambda(r, u)
$$

Thus (3.3.1) gives that $T(r, u)=\lambda(r, u)$, implying that $u$ is rational, a contradiction.

\section{QED}

Remark: We note here that the restriction in Theorem 3.1.1 to $k \geq 2$ is since if $k=1$, then a pole of $f$ will result in a simple zero of $u$, and we do not place any restriction on the number of poles of $f$. 
Proof of Theorem 3.1.2:

Suppose that $u$ is rational. Then $\lambda(r, u)=O(\log r)$, and thus $f^{(k)}$ has only finitely many zeros. Hence by Lemma 3.2.4, $f=R e^{P}$.

Now suppose that $u$ is transcendental. Then there exists no $c \in \mathbb{C}$ such that $\psi \equiv c u^{n}$, since otherwise we have an identity of the form (3.2.14), and produce a contradiction via Lemma 3.2.5. Thus by (3.2.2), (3.2.4) and Lemma 3.2.3,

$$
N^{2+}(r, u)+m(r, u) \leq 2 N_{1}(r, u)+\lambda(r, u)=\lambda(r, u)
$$

Now, a simple pole of $u$ cannot be a pole of $f$, and so must be a zero of $f^{(k)}$. Hence,

$$
T(r, u) \leq \bar{N}\left(r, \frac{1}{f^{(k)}}\right)+N^{2+}(r, u)+m(r, u) \leq \lambda(r, u)
$$

and so $u$ is rational, a contradiction.

Proof of Theorem 3.1.3:

Suppose that $u$ is transcendental, then by Lemma $3.2 .5 \psi / u^{n}$ is non-constant and we apply Lemma 3.2.2 to give $m(r, u)=\lambda(r, u)$. Thus by Lemma 3.2.6, we then have $T(r, u)=\lambda(r, u)$, and so $u$ is not transcendental. Hence assume $u$ is rational, and that $f$ is transcendental. Lemma 3.2.6 then gives us $T(r, f)=O(m(r, u))=\lambda(r, u)=O(\log r)$, a contradiction. Hence $f$ is rational.

QED 


\section{Chapter 4}

\section{A result on more general}

\section{homogeneous differential}

\section{polynomials}

In this chapter, we substantially strengthen an unpublished result of Whitehead from his $\mathrm{PhD}$ thesis [33] using a refinement of his techniques. This work is due to be published in Results in Mathematics [7]. The polynomials used in this chapter are of a more general type than in the previous chapter, although there are still some restrictions.

\subsection{Introduction and result}

Let $M_{j}$ be a differential monomial of the form (1.4.1), in a function $f$, meromorphic in the plane. We consider sums of monomials $M_{j}$ of equal degree $n$, forming homogeneous differential polynomials, and consider what we may deduce about $f$ from knowing properties of this polynomial. 


\section{Theorem 4.1.1}

Let $f$ be nonconstant and meromorphic in the plane. Let $u=f / f^{\prime}, m \in \mathbb{N}$, and let $F$, a homogeneous differential polynomial in $f$ and its derivatives of weight $\Gamma_{F}$, be defined by

$$
F=f^{n}+\sum_{j=1}^{m} a_{j} M_{j}[f] \not \equiv 0,
$$

where the $M_{j}[f]$ are as defined in (1.4.1) with degree $n$, and the $a_{j}$ are small functions with respect to $u$ such that $T\left(r, a_{j}\right)=S(r, u)$. Suppose that

$$
\mu_{0, j} \notin\{n-1, n\} \forall j
$$

and that

$$
\Gamma_{F} \geq 2 n
$$

Then at least one of the following must hold:

1. We have that

$$
f=R e^{P}
$$

for some rational function $R$ and polynomial $P$ in $z$;

2. We have that

$$
F \equiv f^{n}
$$

3. The following inequality holds:

$$
T(r, u) \leq\left(\Gamma_{F}-3\right) \bar{N}_{1}\left(r, \frac{1}{f^{\prime}}\right)+\bar{N}_{2}\left(r, \frac{1}{F}\right)+S(r, u)
$$

where $\bar{N}_{1}\left(r, 1 / f^{\prime}\right)$ counts zeros of $f^{\prime}$ which are not also zeros of $f$, without regard to multiplicity, and $\bar{N}_{2}(r, 1 / F)$ counts zeros of $F$ which are neither poles nor zeros of $f$, again without regard to multiplicity. 
Remark: Conditions (4.1.2) and (4.1.3) force $n \geq 2$ and thus $\Gamma_{F} \geq 4$. Note that this definition of $N_{1}$ is again different to the $N_{1}$ used by Hayman in the statement of the Second Fundamental Theorem (1.3.4), and also different to the $N_{1}$ used in Chapter 3.

This result is an improvement of Whitehead's Theorem 5.16 [33]. Whitehead's result required that there be a term of unique maximal weight (see the remark in the proof of the main theorem for details), replaced condition (4.1.3) with the stricter requirement that there be some $k$ such that $\mu_{0, k}=0$, and had the inequality

$$
T(r, u) \leq \bar{N}\left(r, \frac{1}{f}\right)+\left(\Gamma_{F}-3\right) \bar{N}\left(r, \frac{1}{f^{\prime}}\right)+\bar{N}\left(r, \frac{1}{F}\right)+S(r, u)
$$

in place of (4.1.6).

Condition (4.1.2) is necessary for us to apply this theorem (again, see the remark in the proof of the main theorem for details), since we can construct examples without this assumption such that none of the conclusions of the above theorem hold. For instance, let $F=f^{2}+2 f f^{\prime \prime}-3\left(f^{\prime}\right)^{2}$, with

$$
f=\frac{1}{1-e^{z}}, \quad f^{\prime}=\frac{e^{z}}{\left(1-e^{z}\right)^{2}}, \quad f^{\prime \prime}=\frac{e^{z}\left(1+e^{z}\right)}{\left(1-e^{z}\right)^{3}} .
$$

Then $F=f^{4} \neq f^{2}$, and so conclusion 2 does not hold. Further, neither $F$ nor $f^{\prime}$ have any zeros, and hence conclusion 3 cannot hold. Since conclusion 1 clearly does not hold, our example is such that none of the conclusions hold. Another example using the same $f$ is $F=f^{3}+3 f^{2} f^{\prime \prime}+7\left(f^{\prime}\right)^{3}-3 f f^{\prime} f^{\prime \prime}$.

\section{Corollary 4.1.2}

Let the hypotheses of the main theorem hold, with the additional condition that $m=1$ in (4.1.1) so that $F=f^{n}+a M[f]$ where $a \not \equiv 0$. Then at least one of (4.1.4) and (4.1.6) holds. 
Remark: Whitehead had a similar corollary for his theorem.

\subsection{Lemmas}

\subsubsection{An inequality related to a result of Zhang and $\mathrm{Li}$}

The first part of working towards a proof of the main theorem is to improve a result of Zhang and Li of Tumura-Clunie type. First though, we need a lemma.

\section{Lemma 4.2.1 - Clunie's Lemma $[10]$}

Let $h$ be a transcendental meromorphic function, and let $P[h]$ and $Q[h]$ be polynomials in $h$ and its derivatives with meromorphic functions satisfying $m(r, c)=S(r, h)$ as coefficients. Suppose further that $h^{n} P[h]=Q[h]$, and that $Q[h]$ has degree at most $n$. Then,

$$
m(r, P[h])=S(r, h)
$$

A modified version of this lemma appeared in the previous chapter as Lemma 3.2.1.

\section{Theorem 4.2.2}

Let the function $h$ be non-constant and meromorphic in the plane. Assume that

$$
\psi=h^{n}+P[h] \not \equiv 0,
$$

where $P[h]$ is a differential polynomial in $h$ with coefficients $c_{j}$ which are small functions with respect to $h$, i.e. $T\left(r, c_{j}\right)=S(r, h)$. Suppose that $P[h]$ has degree at most $n-2$. Then at least one of the following is true:

1. We have

$$
T(r, h)<\left(\Gamma_{P}-n+3\right) \bar{N}(r, h)+\bar{N}_{0}\left(r, \frac{1}{\psi}\right)+S(r, h)
$$


as $r \rightarrow \infty$, where $\Gamma_{P}$ is the weight of $P$ and $\bar{N}_{0}(r, 1 / \psi)$ counts zeros of $\psi$ which are not zeros of $h$, without regard to multiplicity;

2. We have

$$
\psi \equiv h^{n} .
$$

Remark: This is an improvement of Whitehead's Theorem 5.8 [33], based on the methods of Zhang and $\mathrm{Li}[36]$, where it makes up the final two pages of their proof, but is not itself presented as a result. Whitehead's version had $\bar{N}(r, 1 / \psi)$ instead of $\bar{N}_{0}(r, 1 / \psi)$. This improvement will be very important later.

We also note here that this result still holds even if $\Gamma_{P}-n+3$ is negative.

Proof:

Differentiating (4.2.2), we get $\psi^{\prime}=n h^{n-1} h^{\prime}+P^{\prime}$, and so

$$
\begin{aligned}
\frac{\psi^{\prime}}{\psi}\left(h^{n}+P\right) & =n h^{n-1} h^{\prime}+P^{\prime}, \\
\frac{\psi^{\prime}}{\psi} P-P^{\prime} & =n h^{n-1} h^{\prime}-\frac{\psi^{\prime}}{\psi} h^{n} \\
& =h^{n-1} H,
\end{aligned}
$$

where

$$
H=n h^{\prime}-\frac{\psi^{\prime}}{\psi} h
$$

\section{Case I:}

Suppose that $H \not \equiv 0$. Then $m\left(r, \psi^{\prime} / \psi\right)=S(r, \psi)$ by the Lemma of the Logarithmic Derivative (1.3.2), and by Lemmas 1.2.2 and 1.3.6, $S(r, \psi)=S(r, h)$. Since the left hand side of (4.2.5) has degree at most $n-2$, we may apply Clunie's Lemma (Lemma 4.2.1), giving that

$$
m(r, H)=S(r, h)
$$


Since $P$ has degree at most $n-2$ and $P^{\prime}$ has at most the same degree, we can write $(4.2 .5)$ as

$$
h^{n-2}(h H)=\frac{\psi^{\prime}}{\psi} P-P^{\prime}
$$

and use Clunie's Lemma (Lemma 4.2.1) to give $m(r, h H)=S(r, h)$. Using this, the First Fundamental Theorem (1.3.1) and (4.2.7), we get

$$
\begin{aligned}
m(r, h) & =m\left(r, \frac{h H}{H}\right) \\
& \leq m\left(r, \frac{1}{H}\right)+m(r, h H) \\
& =T(r, H)-N\left(r, \frac{1}{H}\right)+S(r, h) \\
& =N(r, H)-N\left(r, \frac{1}{H}\right)+S(r, h),
\end{aligned}
$$

and hence

$$
T(r, h) \leq N(r, h)+N(r, H)-N\left(r, \frac{1}{H}\right)+S(r, h)
$$

Let $z_{0} \in \mathbb{C}$, and suppose that $h$ has a pole of order $q \geq 0$ at $z_{0}$, and let its contribution to $n(r, H)-n(r, 1 / H)$ be $-t$. Thus, $t>0$ if $z_{0}$ is a zero of $H$ of multiplicity $t$ and $t<0$ if $z_{0}$ is a pole of $H$ of multiplicity $-t$.

If $q \geq 1$, then a monomial $M=c h^{i_{0}}\left(h^{\prime}\right)^{i_{1}} \ldots\left(h^{(k)}\right)^{i_{k}}$ has a pole of order at most $q i_{0}+(q+1) i_{1}+\ldots+(q+k) i_{k}+s$ where the $s$ is the contribution from the coefficient c. This is

$$
\begin{aligned}
\sum_{j=0}^{k}(j+1) i_{j}+(q-1) \sum_{j=0}^{k} i_{j}+s & =\Gamma_{M}+(q-1) \gamma_{M}+s \\
& \leq \Gamma_{P}+(q-1)(n-2)+S
\end{aligned}
$$

since $\Gamma_{P}$ is the maximum of the $\Gamma_{M}$ over all monomial terms in $P$ and where $S$ is the contribution from the coefficients. We now rewrite (4.2.5) in the form

$$
h^{n-1}=\left(\frac{\psi^{\prime}}{\psi} P-P^{\prime}\right) \frac{1}{H} .
$$


Then we have

$$
(n-1) q \leq \Gamma_{P}+(q-1)(n-2)+S+1+t,
$$

where the 1 comes from $\psi^{\prime} / \psi$ having at most a simple pole, and $t$ is as defined above. Thus,

$$
q-t \leq \Gamma_{P}-n+3+S
$$

and hence $z_{0}$ contributes at most $\Gamma_{P}-n+3+S$ to

$$
n_{1}(r)=n(r, h)+n(r, H)-n(r, 1 / H)
$$

and at least $\Gamma_{P}-n+3$ to

$$
n_{2}(r)=\left(\Gamma_{P}-n+3\right) \bar{n}(r, h)+\bar{n}_{0}\left(r, \frac{1}{\psi}\right)
$$

where $\bar{n}_{0}(r, 1 / \psi)$ counts the distinct points at which $\psi=0$ but $h \neq 0$.

Now suppose that $q=0$ but $t \neq 0$. If $t>0$, then the contribution to $n_{1}(r)$ is negative and the contribution to $n_{2}(r)$ is non-negative. If $t<0$, then $z_{0}$ must be a simple pole of $H$ arising from the term $\psi^{\prime} / \psi$ in (4.2.6). Such a simple pole of $H$ can be caused by a zero of $\psi$ which is not a zero of $h$, which then gives $t=-1$ and contributes 1 to each of $n_{1}(r)$ and $n_{2}(r)$. The only other possibility is a pole of $\psi$ caused by a pole of the coefficients, which contributes 1 to $n_{1}(r)$ and 0 to $n_{2}(r)$, and the number of such poles is $S(r, h)$. Thus (4.2.9) becomes (4.2.3).

\section{Case II:}

Assume now that $H \equiv 0$. Then by (4.2.5), we have $P=\lambda \psi$ for some $\lambda \in \mathbb{C}$. If $\lambda=0$ then $P \equiv 0$ and so (4.2.4) follows by (4.2.2). Now suppose that $\lambda \neq 0$, and let $\Lambda=\lambda^{-1}$. Then we have $h^{n}+P=\psi=\Lambda P$, and so

$$
h^{n-1} h=(\Lambda-1) P .
$$


We apply Clunie's Lemma (Lemma 4.2.1), giving

$$
m(r, h)=S(r, h)
$$

We further note that

$$
h^{2}=(\Lambda-1) P h^{2-n}
$$

and examine the poles of this function. As before, if $h$ has a pole of order $q \geq 1$, then by (4.2.10), $P$ has a pole of order at most $\Gamma_{P}+(q-1)(n-2)+s$, and so $P h^{2-n}$ will have a pole of order at most

$$
\Gamma_{P}+(q-1)(n-2)+s-q(n-2)=\Gamma_{P}-(n-2)+s .
$$

Using (4.2.13),

$$
\begin{aligned}
T(r, h)=N(r, h)+S(r, h) & =\frac{1}{2} N\left(r, h^{2}\right)+S(r, h) \\
& \leq \frac{1}{2}\left(\Gamma_{P}-(n-2)\right) \bar{N}(r, h)+S(r, h) .
\end{aligned}
$$

With $\Gamma_{P}>n-2$, it is easy to see that this implies (4.2.3). If however, $\Gamma_{P} \leq n-2$, then by (4.2.13) and (4.2.14) we have $T(r, h)=S(r, h)$, a contradiction.

QED

\subsubsection{Several lemmas by Whitehead}

We now present several lemmas from [33]. We include the proofs for completeness, as Whitehead's thesis is unpublished. We begin with a result comparing the weight of a monomial with the weight of its derivative. 
Lemma 4.2.3 $[33]$

Let $M[u]$ be a monomial as defined in (1.4.1). If $M[u]$ has weight $\Gamma_{M}$ then $M^{\prime}[u]$ has weight $\Gamma_{M}+1$.

This result is proved by induction on $q$, the highest derivative of $u$ occurring in $M[u]$. We now show that we may write higher derivatives of $f$ in terms of $f$ and $u$.

Lemma 4.2.4 [33]

Let $p \in \mathbb{N}$ and $u=\frac{f}{f^{\prime}}$. Then,

$$
f^{(p)}=f \frac{S_{p}[u]}{u^{p}}
$$

where

$$
\begin{aligned}
& S_{1}[u]=1 \\
& S_{2}[u]=1-u^{\prime}
\end{aligned}
$$

and

$$
S_{p}[u]=\left(1-u^{\prime}\right)\left(1-2 u^{\prime}\right) \ldots\left(1-(p-1) u^{\prime}\right)+u T_{p-2}[u]
$$

for $p \geq 3$, with $T_{p-2}[u]$ a differential polynomial in $u$ with constant coefficients and degree at most $p-2$, such that $T_{0}[u] \equiv 0$. Further, each $S_{p}[u]$ has degree $p-1$ and weight $2(p-1)$.

Proof:

We begin by noting that

$$
f^{\prime}=\frac{f}{u}=\frac{f}{u} S_{1}[u] \quad \text { and } \quad f^{\prime \prime}=\frac{f^{\prime}}{u}-\frac{f u^{\prime}}{u^{2}}=\frac{f}{u^{2}} S_{2}[u]
$$


and thus the lemma holds for $p \in\{1,2\}$. Assume it holds for some $p=k \geq 2$, then we have

$$
\frac{f^{(k)}}{f}=\frac{S_{k}[u]}{u^{k}}
$$

and hence

$$
\begin{aligned}
\frac{f^{(k+1)}}{f} & =\left(\frac{f^{(k)}}{f}\right)^{\prime}+\frac{f^{(k)}}{f} \frac{f^{\prime}}{f} \\
& =\left(\frac{S_{k}[u]}{u^{k}}\right)^{\prime}+\frac{S_{k}[u]}{u^{k}} \frac{1}{u} \\
& =\frac{S_{k+1}[u]}{u^{k+1}},
\end{aligned}
$$

where

$$
S_{k+1}[u]=u S_{k}^{\prime}[u]+\left(1-k u^{\prime}\right) S_{k}[u]
$$

We now prove (4.2.16). Substituting into (4.2.17), we have

$$
S_{k+1}[u]=\left(1-u^{\prime}\right) \ldots\left(1-(k-1) u^{\prime}\right)\left(1-k u^{\prime}\right)+\left(1-k u^{\prime}\right) u T_{k-2}[u]+u S_{k}^{\prime}[u],
$$

and we set

$$
T_{k-1}[u]=\left(1-k u^{\prime}\right) T_{k-2}[u]+S_{k}^{\prime}[u]
$$

which has degree at most

$$
\max \{1+(k-2), k-1\}=k-1=(k+1)-2 .
$$

Also, (4.2.17) and Lemma 4.2.3 show that $S_{k+1}[u]$ has weight at most

$$
\max \left\{\Gamma_{S_{k}}+2, \Gamma_{S_{k}}, \Gamma_{S_{k}}+2\right\}=2(k-1)+2=2((k+1)-1),
$$

and the presence of the term $\left(1-u^{\prime}\right) \ldots\left(1-k u^{\prime}\right)$ in $S_{k+1}[u]$ shows that the degree of $S_{k+1}[u]$ is $(k+1)-1$ and the weight is $2((k+1)-1)$. 
We now show that we may write a differential monomial in $f$ in terms of $f$ and a differential polynomial in $u$.

\section{Lemma 4.2.5 $[33]$}

Let the hypotheses of Theorem 4.1.1 hold, let $L=\Gamma_{F}-n$ and $u=\frac{f}{f^{\prime}}$. Then

$$
u^{L} \frac{M_{j}[f]}{f^{n}}=V_{j}[u]
$$

where $V_{j}[u]$ is a differential polynomial in $u$ with constant coefficients, of degree at most $L-2$ and weight at most $2 \Gamma_{F}-n-6$.

\section{Proof:}

By the hypotheses, $\Gamma_{F} \geq 2 n$, and so $L \geq n$. We apply Lemma 4.2.4 to (1.4.1),

$$
\begin{aligned}
u^{L} \frac{M_{j}[f]}{f^{n}} & =u^{L} \prod_{p=1}^{q}\left(\frac{f^{(p)}}{f}\right)^{\mu_{p, j}} \\
& =u^{L} \prod_{p=1}^{q}\left(\frac{S_{p}[u]}{u^{p}}\right)^{\mu_{p, j}} \\
& =u^{\delta_{j}} \prod_{p=1}^{q} S_{p}[u]^{\mu_{p, j}} \\
& =V_{j}[u],
\end{aligned}
$$

where

$$
\begin{aligned}
\delta_{j} & =L-\sum_{p=0}^{q} p \mu_{p, j} \\
& =\Gamma_{F}-n-\sum_{p=0}^{q} p \mu_{p, j} \\
& =\Gamma_{F}-\sum_{p=0}^{q}(p+1) \mu_{p, j} \\
& =\Gamma_{F}-\Gamma_{j} \\
& \geq 0 .
\end{aligned}
$$


Since $\mu_{0, j} \notin\{n-1, n\}$, we have $\mu_{1, j}+\ldots+\mu_{q, j} \geq 2$. Thus using Lemma 4.2.4

$$
\begin{aligned}
\gamma_{V_{j}} & \leq \delta_{j}+\sum_{p=1}^{q}(p-1) \mu_{p, j} \\
& =L-\sum_{p=1}^{q}\left(p \mu_{p, j}-(p-1) \mu_{p, j}\right) \\
& =L-\sum_{p=1}^{q} \mu_{p, j} \\
& \leq L-2 .
\end{aligned}
$$

Further, again by Lemma 4.2.4,

$$
\begin{aligned}
\Gamma_{V_{j}} & \leq \delta_{j}+2 \sum_{p=1}^{q}(p-1) \mu_{p, j} \\
& =L-\sum_{p=1}^{q} p \mu_{p, j}+2 \sum_{p=1}^{q}(p-1) \mu_{p, j} \\
& =L+\sum_{p=1}^{q}(p+1) \mu_{p, j}-3 \sum_{p=1}^{q} \mu_{p, j} \\
& \leq L+\Gamma_{j}-6 \\
& =\Gamma_{F}-n+\Gamma_{j}-6 \\
& \leq 2 \Gamma_{F}-n-6 .
\end{aligned}
$$

\subsubsection{Some final lemmas}

We conclude this section with two lemmas which are improvements of results from Whitehead's thesis [33].

\section{Lemma 4.2.6}

We have

$$
\bar{N}(r, u) \leq \bar{N}_{1}\left(r, \frac{1}{f^{\prime}}\right)
$$

where the right hand term counts zeros of $f^{\prime}$ which are not also zeros of $f$, without regard to multiplicity. 
Proof:

Since $u=f / f^{\prime}$, all poles of $u$ must come from poles of $f$ or zeros of $f^{\prime}$. But a pole of $f$, or a zero of $f^{\prime}$ which is also a zero of $f$, would result in a zero of $u$. Therefore all poles of $u$ must come from zeros of $f^{\prime}$ which are not also zeros of $f$.

QED

\section{Lemma 4.2.7}

Let $F$ be as defined in (4.1.1), and $L=\Gamma_{F}-n$ as before. Further, let

$$
\psi=\frac{u^{L} F}{f^{n}} .
$$

Then we have

$$
\bar{N}_{0}\left(r, \frac{1}{\psi}\right)=\bar{N}_{2}\left(r, \frac{1}{F}\right)
$$

where $\bar{N}_{0}(r, 1 / \psi)$ counts the zeros of $\psi$ which are not zeros of $u$ without regard to multiplicity, and $\bar{N}_{2}(r, 1 / F)$ counts zeros of $F$ which are neither poles nor zeros of $f$, again without regard to multiplicity.

Proof:

$\psi$ could have a zero if $f$ has a pole, if $F$ has a zero or if $u$ has a zero. However since $u=f / f^{\prime}$, any pole or zero of $f$ is also a zero of $u$ and thus is not counted by $\bar{N}_{0}(r, 1 / \psi)$. 


\subsection{Proof of Theorem 4.1.1}

If $u$ is rational, then by Lemma 1.4.3 we obtain the first conclusion (4.1.4). Suppose now that $u$ is transcendental. Then by (4.1.1) and Lemma 4.2.5,

$$
\psi=\frac{u^{L} F}{f^{n}}=u^{L}+\sum_{j=1}^{m} a_{j} u^{L} \frac{M_{j}[f]}{f^{n}}=u^{L}+\sum_{j=1}^{m} a_{j} V_{j}[u] .
$$

Since $V_{j}[u]$ has constant coefficients and degree at most $L-2$, we may apply Theorem 4.2.2; and so either $\psi \equiv u^{L}$, and thus $F \equiv f^{n}$; or

$$
T(r, u)<\left(\max \left\{\Gamma_{V_{j}}\right\}-L+3\right) \bar{N}(r, u)+\bar{N}_{0}\left(r, \frac{1}{\psi}\right)+S(r, u) .
$$

Thus, by Lemma 4.2.5,

$$
\begin{aligned}
T(r, u) & <\left(2 \Gamma_{F}-n-6-L+3\right) \bar{N}(r, u)+\bar{N}_{0}\left(r, \frac{1}{\psi}\right)+S(r, u) \\
& =\left(\Gamma_{F}-3\right) \bar{N}(r, u)+\bar{N}_{0}\left(r, \frac{1}{\psi}\right)+S(r, u),
\end{aligned}
$$

from which (4.1.6) follows by Lemmas 4.2.6 and 4.2.7.

Remark: Whitehead's requirement that there be a term of unique maximal weight came from his version of Lemma 4.2.7, which did not ignore zeros of $u$. He noted that having a pole of $f$ with two monomials of maximal weight could allow one to cancel the other out. However, since we ignore zeros of $u$, and any pole of $f$ is a zero of $u$, we may safely ignore poles of $f$, and so this requirement can be disregarded.

The requirement (4.1.2) stems from the hypotheses of Theorem 4.2.2. If (4.1.2) does not hold, then we could have that

$$
\mu_{1, j}+\ldots+\mu_{q, j}=1
$$

which in Lemma 4.2.5 would give that $V_{j}[u]$ could have degree $L-1$, and so we would not be able to apply Theorem 4.2.2 in the above proof. 


\subsubsection{Proof of Corollary 4.1.2}

Using the main theorem, at least one of (4.1.4), (4.1.5) or (4.1.6) holds. Suppose that (4.1.5) holds, then

$$
a \prod_{p=1}^{q}\left(f^{(p)}\right)^{\mu_{p}} \equiv 0,
$$

and so $f^{(p)} \equiv 0$ for some $1 \leq p \leq q$, since $a \not \equiv 0$. Thus $f$ is a polynomial and so satisfies (4.1.4).

QED

Remark: Whitehead proved a version of this for his theorem, the method is identical. 


\section{Chapter 5}

\section{A normal families result for}

\section{homogeneous differential}

\section{polynomials}

In this chapter, we use a result of Tumura-Clunie type from the previous chapter and show that for a homogeneous differential operator acting on a family $\mathcal{F}$ of functions $f$ with certain restrictions on zeros, there exist sufficient conditions such that the family of functions $\mathcal{U}=\left\{f / f^{\prime}: f \in \mathcal{F}\right\}$ is normal.

\subsection{Introduction and result}

Let $\mathcal{H}$ be a family of meromorphic functions. We say that $\mathcal{H}$ is normal if every sequence of functions $\left(h_{n}\right) \subseteq \mathcal{H}$ has a subsequence which converges locally uniformly as $n \rightarrow \infty$, possibly to infinity. For example, the family of functions $\left\{f_{n}(z)=z+n\right\}$ is normal, while the family $\left\{f_{n}(z)=z^{n}\right\}$ is not normal. A classic result in this field is Montel's Theorem, which states that if a family of holomorphic functions on a domain all omit 
the same two values $a, b \in \mathbb{C}$, that family is normal. Normal families have been the subject of much study, overviews of which can be found in [2] and [35]. However, the references to differential polynomials in these works are mainly to exceptional values of certain very specific polynomials in $f$ and $f^{\prime}$. Bergweiler does note in [2] the following result by himself and Langley, which gives conditions such that the family of logarithmic derivatives is normal:

\section{Proposition 5.1.1 [3]}

Let $k \geq 2$, and let $\mathcal{F}$ be a family of functions meromorphic on a domain D. Suppose that $f$ and $f^{(k)}$ have no zeros in $D$ for all $f \in \mathcal{F}$. Then the family $\left\{f^{\prime} / f: f \in \mathcal{F}\right\}$ is normal.

The example $f_{n}(z)=e^{n z}$, for which all derivatives are zero-free, makes it clear that the conclusion in Prop. 5.1.1, that the family of logarithmic derivatives is normal, cannot be replaced by a conclusion that the family of functions themselves is normal. The result which we prove below gives more conditions such that the family of logarithmic derivatives is normal, in particular by application of certain homogeneous differential polynomials $F$, and with restrictions on the zeros of $f^{\prime}$ and $F$. We note that unlike in the Bergweiler-Langley result above, we do not require that either $f$ or any derivative of $f$ be nonvanishing.

\section{Theorem 5.1.2}

Let the homogeneous differential polynomial $F[f]$, of degree $n$ and weight $\Gamma_{F}$, be 
defined by

$$
F[f]=f^{n}+\sum_{j=1}^{d} c_{j} M_{j}[f],
$$

where the $M_{j}[f]$ are as defined in (1.4.1), and the $c_{j}$ are non-zero constants. Suppose that

$$
\mu_{0, j} \notin\{n-1, n\} \forall j
$$

and that

$$
\Gamma_{F} \geq 2 n
$$

For each $j$, define

$$
\alpha_{j}=\frac{\mu_{2, j}+2 \mu_{3, j}+\ldots+(k-1) \mu_{k, j}}{\mu_{1, j}+\mu_{2, j}+\ldots+\mu_{k, j}}
$$

Let $\mathcal{F}$ be a family of non-constant meromorphic functions $f$ on a domain $D$, with the property that, for all $f \in \mathcal{F}, f^{\prime}=0$ only if $f=0$, and that $F[f]=0$ only if $f \in\{0, \infty\}$.

If there exists some unique $j$ which has maximal $\alpha_{j}$; or if for all $j$, we have $\mu_{l, j}=0$ for all $l>2$; then the family $\mathcal{U}=\left\{f / f^{\prime}: f \in \mathcal{F}\right\}$ is normal, and so is the family of logarithmic derivatives $\left\{f^{\prime} / f: f \in \mathcal{F}\right\}$.

Remark: The properties of $\mathcal{F}$ are guided by (4.2.3), in order that it lead to a contradiction. The two conditions on the form of the differential polynomial $F$ are due to the conditions of Theorem 4.2.2, and the condition that the $c_{j}$ be constant is simply for ease of use, as normal families results become arduous when using non-constant coefficients. We also note that the properties of $\mathcal{F}$ mean that $\mathcal{U}$ is a family of holomorphic functions, since $f^{\prime}=0$ only if $f=0$. 


\subsection{Lemmas}

We first state the Pang-Zalcman Lemma [35], one of the most useful results in the study of normal families.

\section{Lemma 5.2.1 - The Pang-Zalcman Lemma [35]}

Let $\mathcal{U}$ be a family of functions meromorphic on a domain $D \subseteq \mathbb{C}$, let $\beta, \gamma \in \mathbb{N}, \alpha \in \mathbb{R}$ with $-\beta<\alpha<\gamma$. Suppose that all functions in $\mathcal{U}$ have no zeros with multiplicity lower than $\beta$ and no poles with multiplicity lower than $\gamma$. Further suppose that $\mathcal{U}$ is not normal at $z_{0} \in D$. Then there exist a sequence $\left(u_{n}\right)$ in $\mathcal{U}$, a sequence $\left(z_{n}\right)$ in $D$, a sequence $\rho_{n}$ of positive real numbers and a nonconstant meromorphic function $v$ such that $z_{n} \rightarrow z_{0}$, $\rho_{n} \rightarrow 0$ and

$$
\rho_{n}^{\alpha} u_{n}\left(z_{n}+\rho_{n} z\right) \rightarrow v(z)
$$

locally uniformly in $\mathbb{C}$.

We now prove an equivalence between the limit of a function of $f_{m}$ and certain differential polynomials.

\section{Lemma 5.2.2}

Let the $f_{m}$ be meromorphic functions on a domain D, members of a family of functions $\mathcal{F}$ with the property that $f_{m}^{\prime}=0$ only if $f_{m}=0$. Let $\rho_{m} \rightarrow 0$ from above as $m \rightarrow \infty$, and let $\left(z_{m}\right)$ be a sequence of points tending to a limit $z_{0} \in D$ as $m \rightarrow \infty$. Define

$$
v_{m}(z)=\rho_{m}^{\alpha} \frac{f_{m}\left(z_{m}+\rho_{m} z\right)}{f_{m}^{\prime}\left(z_{m}+\rho_{m} z\right)}
$$

for some fixed $\alpha>-1$, and let $v_{m} \rightarrow v$ locally uniformly on $\mathbb{C}$ as $m \rightarrow \infty$. Define, for 
$p \in \mathbb{N}$,

$$
\phi_{p, m}(z)=\rho_{m}^{p-1-\alpha} v_{m}^{p}(z) \frac{f_{m}^{(p)}\left(z_{m}+\rho_{m} z\right)}{f_{m}\left(z_{m}+\rho_{m} z\right)}
$$

Then, as $m \rightarrow \infty$,

$$
\phi_{p, m} \rightarrow \Delta_{p}
$$

locally uniformly on $\mathbb{C}$, where

$$
\Delta_{p}=v^{p}\left(\frac{1}{v}\right)^{(p-1)}
$$

Proof:

Note first that $\phi_{1, m}=1=\Delta_{1}$. Note also that $\phi_{p, m}$ is analytic, since a pole of $f_{m}^{(p)} / f_{m}$ has multiplicity $p$ and so is cancelled out by a zero of multiplicity $p$ generated by $v_{m}^{p}=\left(\rho_{m}^{\alpha} f_{m} / f_{m}^{\prime}\right)^{p}$. We now show that if (5.2.3) holds for some $p=q \in \mathbb{N}$, then it holds for $p=q+1$. We may write

$$
\rho_{m}^{q-1-\alpha} \frac{f_{m}^{(q)}}{f_{m}}=v_{m}^{-q} \phi_{q, m}
$$

where $f_{m}$ and its derivatives are evaluated at $z_{m}+\rho_{m} z$, and all other terms are evaluated at $z$. Differentiating, we have

$$
\rho_{m}^{q-1-\alpha+1}\left(\frac{f_{m}^{(q+1)}}{f_{m}}-\frac{f_{m}^{(q)}}{f_{m}} \frac{f_{m}^{\prime}}{f_{m}}\right)=-q v_{m}^{-q-1} v_{m}^{\prime} \phi_{q, m}+v_{m}^{-q} \phi_{q, m}^{\prime}
$$

Multiplying through by $v_{m}^{q+1}(z)$ we get

$$
\rho_{m}^{(q+1)-1-\alpha} v_{m}^{q+1}\left(\frac{f_{m}^{(q+1)}}{f_{m}}-\frac{f_{m}^{(q)}}{f_{m}} \frac{f_{m}^{\prime}}{f_{m}}\right)=-q v_{m}^{\prime} \phi_{q, m}+v_{m} \phi_{q, m}^{\prime},
$$

and then rearranging we have

$$
\begin{aligned}
\rho_{m}^{(q+1)-1-\alpha} v_{m}^{q+1} \frac{f_{m}^{(q+1)}}{f_{m}} & =\rho_{m}^{(q+1)-1-\alpha} v_{m}^{q+1} \frac{f_{m}^{(q)}}{f_{m}} \frac{f_{m}^{\prime}}{f_{m}}-q v_{m}^{\prime} \phi_{q, m}+v_{m} \phi_{q, m}^{\prime} \\
\phi_{q+1, m} & =\left(\rho_{m}^{q-1-\alpha} v_{m}^{q} \frac{f_{m}^{(q)}}{f_{m}}\right) \rho_{m}^{1+\alpha}\left(\rho_{m}^{-\alpha} v_{m} \frac{f_{m}^{\prime}}{f_{m}}\right)-q v_{m}^{\prime} \phi_{q, m}+v_{m} \phi_{q, m}^{\prime} \\
& =\rho_{m}^{1+\alpha} \phi_{q, m} \phi_{1, m}-q v_{m}^{\prime} \phi_{q, m}+v_{m} \phi_{q, m}^{\prime} .
\end{aligned}
$$


We take the limit as $m \rightarrow \infty$, which, since $1+\alpha>0$, gives that

$$
\begin{aligned}
\phi_{q+1, m} & \rightarrow 0 . \Delta_{q} \cdot 1-q v^{\prime} \Delta_{q}+v \Delta_{q}^{\prime} \\
& =v \Delta_{q}^{\prime}-q v^{\prime} \Delta_{q} \\
& =v\left(v^{q}\left(\frac{1}{v}\right)^{(q-1)}\right)^{\prime}-q v^{\prime} v^{q}\left(\frac{1}{v}\right)^{(q-1)} \\
& =v^{q+1}\left(\frac{1}{v}\right)^{(q)}+q v^{q} v^{\prime}\left(\frac{1}{v}\right)^{(q-1)}-q v^{q} v^{\prime}\left(\frac{1}{v}\right)^{(q-1)} \\
& =\Delta_{q+1}
\end{aligned}
$$

as required. Thus by induction, we achieve the result.

Recalling Theorem 4.2.2, we are in a position to prove Theorem 5.1.2.

\subsection{Proof of Theorem 5.1.2}

First, let $\alpha$ be the maximum of the $\alpha_{j}$, defined by (5.1.3), over all monomials occurring in $F[f]$. It is clear to see from the definition that $\alpha \geq 0$. We now proceed by contradiction. Suppose that $\mathcal{U}$ is not normal. Then by the Pang-Zalcman Lemma, given some $\alpha^{\prime} \in$ $(-\beta, \gamma)$, there exist a sequence $\left(u_{m}\right) \subseteq \mathcal{U}$, a sequence $\left(z_{m}\right) \rightarrow z_{0}$ in $D$, and a sequence of positive real numbers $\rho_{m} \rightarrow 0$ such that

$$
\rho_{m}^{\alpha} u_{m}\left(z_{m}+\rho_{m} z\right)=v_{m}(z)
$$

tends locally uniformly on $\mathbb{C}$ to a non-constant function $v(z)$. Because $\mathcal{U}$ is a family of holomorphic functions, we have that $\gamma=\infty$, and so in particular this holds for any $\alpha^{\prime} \geq 0$, and so we may choose $\alpha^{\prime}=\alpha$. We also have that $v$ is entire, since for any $f_{m} \in \mathcal{F}, f_{m}^{\prime}=0$ only if $f_{m}=0$ and the $u_{m}$ are holomorphic. 
Define $F_{m}=F\left[f_{m}\right]$ and let $M_{j}\left[f_{m}\right]=\left(f_{m}\right)^{\mu_{0, j}}\left(f_{m}^{\prime}\right)^{\mu_{1, j}} \ldots\left(f_{m}^{(k)}\right)^{\mu_{k, j}}$ be a differential monomial in $f_{m}$ of degree $n$, weight $\Gamma_{j}$ and $\alpha$-value $\alpha_{j}$ as defined by (5.1.3). Let

$$
\Psi_{m}=\frac{v_{m}^{L} F_{m}}{f_{m}^{n}}
$$

where $\Psi_{m}$ and $v_{m}$ are evaluated at $z, f_{m}$ and $F_{m}$ at $z_{m}+\rho_{m} z$, and $L=\Gamma_{F}-n$. Then the term of $\Psi_{m}$ generated by $M_{j}\left[f_{m}\right]$ is

$$
v_{m}^{L}\left(\frac{f_{m}^{\prime}}{f_{m}}\right)^{\mu_{1, j}} \ldots\left(\frac{f_{m}^{(k)}}{f_{m}}\right)^{\mu_{k, j}}=\rho_{m}^{A_{j}} v_{m}^{\Gamma_{F}-\Gamma_{j}}\left(\rho_{m}^{-\alpha} v_{m} \frac{f_{m}^{\prime}}{f_{m}}\right)^{\mu_{1, j}} \ldots\left(\rho_{m}^{k-1-\alpha} v_{m}^{k} \frac{f_{m}^{(k)}}{f_{m}}\right)^{\mu_{k, j}}
$$

where, using (5.1.3),

$$
\begin{aligned}
A_{j} & =\alpha \mu_{1, j}+(\alpha-1) \mu_{2, j}+\ldots+(\alpha-k+1) \mu_{k, j} \\
& =\alpha\left(\mu_{1, j}+\mu_{2, j}+\ldots+\mu_{k, j}\right)-\left(\mu_{2, j}+2 \mu_{3, j}+\ldots+(k-1) \mu_{k, j}\right) \\
& =\left(\mu_{1, j}+\mu_{2, j}+\ldots+\mu_{k, j}\right)\left(\alpha-\frac{\mu_{2, j}+2 \mu_{3, j}+\ldots+(k-1) \mu_{k, j}}{\mu_{1, j}+\mu_{2, j}+\ldots+\mu_{k, j}}\right) \\
& =\left(n-\mu_{0, j}\right)\left(\alpha-\alpha_{j}\right) \geq 0 .
\end{aligned}
$$

It is clear to see that this is, with the notation of Lemma 5.2.2,

$$
v_{m}^{\Gamma_{F}-\Gamma_{j}}\left(\phi_{1, m}\right)^{\mu_{1, j}}\left(\phi_{2, m}\right)^{\mu_{2, j}} \ldots\left(\phi_{k, m}\right)^{\mu_{k, j}} \rho_{m}^{A_{j}}
$$

and thus

$$
\Psi_{m}=v^{L}+\sum_{j} \rho_{m}^{A_{j}} v_{m}^{\Gamma_{F}-\Gamma_{j}}\left(\phi_{1, m}\right)^{\mu_{1, j}}\left(\phi_{2, m}\right)^{\mu_{2, j}} \ldots\left(\phi_{k, m}\right)^{\mu_{k, j}}
$$

Although we can apply Lemma 5.2.2 for any $\alpha>-1$, it is more convenient to consider the case $\alpha=0$ using standard methods as follows. This value of $\alpha$ corresponds to the case that $F$ is a polynomial in $f$ and $f^{\prime}$ only, and thus the differential polynomial $F_{m}$ has the form

$$
F_{m}=f_{m}^{n}+\sum_{j=0}^{n-2} c_{j}\left(f_{m}\right)^{j}\left(f_{m}^{\prime}\right)^{n-j}
$$


where at least one $c_{j}$ is non-zero. Let $u_{m}=f_{m} / f_{m}^{\prime}$, then we may rewrite

$$
\begin{aligned}
\psi_{m} & =\frac{u_{m}^{L} F_{m}}{f_{m}^{n}}=u_{m}^{L}+\sum_{j=0}^{n-2} c_{j} u_{m}^{L+j-n} \\
& =\left(\rho_{m}^{-\alpha} v_{m}\right)^{L}+\sum_{j=0}^{n-2} c_{j}\left(\rho_{m}^{-\alpha} v_{m}\right)^{L+j-n}
\end{aligned}
$$

where $\psi_{m}$ and $v_{m}$ are evaluated at $z, F_{m}, u_{m}$ and $f_{m}$ at $z_{m}+\rho_{m} z$. Taking the limit, since $\alpha=0$, we have

$$
\psi_{m} \rightarrow \Psi=v^{L}+\sum_{j=0}^{n-2} c_{j} v^{L+j-n},
$$

where $\Psi$ is non-constant because $v$ is non-constant. Suppose that $w_{0} \in \mathbb{C}$ is a zero of $\Psi$ but not of $v$. Then Hurwitz's theorem gives, for large $m$, a zero $w_{m}$ of $\psi_{m}$ which is close to $w_{0}$ but not a zero of $v_{m}$, and so $z_{m}+\rho_{m} w_{m}$ is a zero of $F_{m}$, since if it were a pole or a zero of $f_{m}$ then $w_{0}$ would be a zero of $v_{m}$. Since all zeros of $F_{m}$ are zeros or poles of $f_{m}$, this forces $v_{m}\left(w_{m}\right)=0$, a contradiction. Thus, all zeros of $\Psi$ are also zeros of $v$, and thus when we apply Theorem 4.2.2 to $\Psi$ the inequality (4.2.3) fails, and so we have

$$
\sum_{j=0}^{n-2} c_{j} v^{L+j-n} \equiv 0
$$

which is only solved by constants. This violates the conclusion of the Pang-Zalcman Lemma that the function $v$ is non-constant.

We may now assume that $\alpha>0$. Taking the limit of (5.3.2) as $m \rightarrow \infty$, we have

$$
\psi_{m} \rightarrow \Psi=v^{L}+\ldots+c_{j} v^{\Gamma_{F}-\Gamma_{j}}\left(\Delta_{1}\right)^{\mu_{1, j}}\left(\Delta_{2}\right)^{\mu_{2, j}} \ldots\left(\Delta_{k}\right)^{\mu_{k, j}} \beta_{j}+\ldots=v^{L}+\Phi
$$

where $\rho_{m}^{A_{j}} \rightarrow \beta_{j} \in\{0,1\}$. As we saw in the proof of Lemma 5.2.2, $\Delta_{q+1}=v \Delta_{q}^{\prime}-q v^{\prime} \Delta_{q}$. Thus, it is clear that since $\Delta_{1}=1$, each $\Delta_{q}$ will have degree at most $q-1$. Thus we 
have

$$
\begin{aligned}
\operatorname{deg}(\Phi) & \leq \max \left\{\Gamma_{F}-\Gamma_{j}+\mu_{2, j}+2 \mu_{3, j}+\ldots+(k-1) \mu_{k, j}\right\} \\
& =\max \left\{L-\Gamma_{j}+\mu_{0, j}+\mu_{1, j}+2 \mu_{2, j}+\ldots+k \mu_{k, j}\right\} \\
& =\max \left\{L-\left(\mu_{1, j}+\mu_{2, j}+\ldots+\mu_{k, j}\right)\right\} \\
& \leq L-2
\end{aligned}
$$

by the hypotheses of the theorem and the fact that $L=\Gamma_{F}-n$.

Consider first the case $\Psi \equiv 0$. Since $v$ is entire, we have two cases: $v$ is polynomial, or $v$ is transcendental. Assume first that $v$ is a polynomial of degree $q$, then $\Phi$ is a polynomial of degree at most $q(L-2)$, but $v^{L}$ is a polynomial of degree $q L$, and thus we have a contradiction unless $q=0$, in which case $v$ is constant, which violates the Pang-Zalcman Lemma. Now consider $v$ to be transcendental. We write $v^{L-2} v^{2}=-\Phi$, and thus by Clunie's Lemma (Lemma 4.2.1) return $m\left(r, v^{2}\right)=2 m(r, v)=S(r, v)$, which contradicts our statement that $v$ is entire. Hence $\Psi \not \equiv 0$, and we may apply Theorem 4.2.2. This gives us that either that

$$
\Psi \equiv v^{L} \quad \text { and so } \quad \Phi \equiv 0
$$

or

$$
T(r, v)<\left(\Gamma_{\Phi}-L+3\right) \bar{N}(r, v)+\bar{N}_{0}\left(r, \frac{1}{\Psi}\right)+S(r, v)
$$

where $\Gamma_{\Phi}$ is the weight of $\Phi$ and $\bar{N}_{0}(r, 1 / \Psi)$ counts zeros of $\Psi$ which are not zeros of $v$, without regard to multiplicity. We showed earlier that $v$ is entire, hence $\bar{N}(r, v)=0$. Now consider zeros of $\Psi$. Since $\Psi \not \equiv 0$, if $\Psi$ has a zero at some point $w_{0}$, then $\Psi$ must be non-constant, and thus, since $\psi_{m} \rightarrow \Psi$, Hurwitz's Theorem gives a zero $w_{m}$ of $\psi_{m}$, with $w_{m} \rightarrow w_{0}$ as $m \rightarrow \infty$. Therefore, by (5.3.1), we have that $w_{m}$ is a zero of $v_{m}$, in which case we do not count it; or $z_{m}+\rho_{m} w_{m}$ is a pole of $f$ or a zero of $F_{m}$, and so is a zero of 
$u_{m}$ by the properties of $\mathcal{F}$ and $\mathcal{U}$, and therefore $w_{m}$ is a zero of $v_{m}$ and is again ignored. Thus $w_{0}$ makes no addition to $\bar{N}_{0}(r, 1 / \Psi)$, and so $\bar{N}_{0}(r, 1 / \Psi) \equiv 0$. Thus the inequality fails and we have that $\Phi \equiv 0$.

We now consider the first case given in the theorem, that there is a unique term $M_{j}\left[f_{m}\right]$ with maximal $\alpha_{j}$. Since $A_{j}=\left(n-\mu_{0, j}\right)\left(\alpha-\alpha_{j}\right)$, it is clear that $A_{j}=0$ if $\alpha_{j}=\alpha$, and is otherwise positive since for any monomial $M_{j}, n-\mu_{j, 0} \geq 2$ by the conditions of the theorem. Since $\rho_{m}^{A_{j}} \rightarrow \beta_{j}$ and $\rho_{m} \rightarrow 0$, it is clear that $\beta_{j}=1$ if $A_{j}=0$, and if $A_{j}>0$ then $\beta_{j}=0$, and thus that particular term vanishes. Therefore, since we choose $\alpha$ to be the maximal $\alpha_{j}$, and that $\alpha_{j}$ is unique, we have only a single term remaining:

$$
\Phi=c_{j} v^{\Gamma_{F}-\Gamma_{j}}\left(\Delta_{1}\right)^{\mu_{1, j}}\left(\Delta_{2}\right)^{\mu_{2, j}} \ldots\left(\Delta_{k}\right)^{\mu_{k, j}} \equiv 0
$$

and so one of the following is true:

1. $c_{j}=0$

2. $v \equiv 0$

3. $\Delta_{p} \equiv 0$ for some $p \in \mathbb{N}$.

Case 1 is ruled out by the hypotheses of the theorem. Case 2 violates the statement of the Pang-Zalcman Lemma that $v$ is non-constant. Case 3 implies that either $v \equiv 0$, which we have ruled out, or that $(1 / v)^{(p-1)} \equiv 0$, which implies that $(1 / v)$ is a polynomial, and hence either $v$ is constant, which again violates Pang-Zalcman, or $v$ is not entire, which is again a contradiction.

Finally, we come to the second case of the theorem, that there are no derivatives in $F$ of higher order than $f^{\prime \prime}$, and, since we have dealt with the case $\alpha=0$, there is at least one term containing $f^{\prime \prime}$. We have $\alpha=\mu_{2, j} /\left(\mu_{1, j}+\mu_{2, j}\right)$ for some $j$, and this value of $\alpha_{j}$ is 
shared by at least two monomials (otherwise, see the previous case). Thus $\mu_{1, j}=\gamma \mu_{2, j}$ where $\gamma=\alpha^{-1}-1$. Then

$$
\begin{aligned}
\Phi & =\sum_{j: A_{j}=0} c_{j} v^{L-\mu_{1, j}-2 \mu_{2, j}} \Delta_{1}^{\mu_{1, j}} \Delta_{2}^{\mu_{2, j}} \\
& =\sum_{j: A_{j}=0} c_{j} v^{L-(\gamma+2) \mu_{2, j}}(1)^{\gamma \mu_{2, j}}\left(-v^{\prime}\right)^{\mu_{2, j}} \\
& =v^{L} \sum_{j: A_{j}=0} c_{j}(-1)^{\mu_{2, j}}\left(\frac{v^{\prime}}{v^{\gamma+2}}\right)^{\mu_{2, j}}
\end{aligned}
$$

By our earlier working, we know $\Phi \equiv 0$, and hence we have that either $v^{L} \equiv 0$, which contradicts the Pang-Zalcman Lemma, or that there exists a polynomial $Q$ such that

$$
Q\left[\frac{v^{\prime}}{v^{\gamma+2}}\right] \equiv 0
$$

which implies that $v^{\prime} \equiv \lambda_{1} v^{\gamma+2}$ for some constant $\lambda_{1}$. Thus, since $\gamma \neq-1$,

$$
\begin{aligned}
\int v^{-\gamma-2} d v & =\int \lambda_{1} d z \\
v^{-\gamma-1} & =\lambda_{2} z+\lambda_{3}
\end{aligned}
$$

for constants $\lambda_{p}$, and therefore $v=\left(\lambda_{2} z+\lambda_{3}\right)^{-1 /(\gamma+1)}$. Clearly this will only be nonconstant entire when $-1 /(\gamma+1) \in \mathbb{N}$. This in turn, by the definition of $\gamma$, gives us that $-\alpha \in \mathbb{N}$, which is clearly impossible since $\alpha>0$. Therefore $v$ is not entire, and so violates the Pang-Zalcman lemma.

Thus, we see that in all cases, we reach a contradiction of the Pang-Zalcman Lemma, and hence $\mathcal{U}$ is normal. Hence, since a subsequence in a normal family may converge to $\infty$, the family of reciprocals of $\mathcal{U}$, the family of logarithmic derivatives of $\mathcal{F}$, is also normal.

QED

Remark: We note here that this method can be applied to any homogeneous differential polynomial of the form given in the theorem, not necessarily just those with no deriva- 
tives higher than $f^{\prime \prime}$ or a unique maximal $\alpha_{j}$. However, these polynomials give rise to differential equations of the form

$$
\Phi=\sum_{j} c_{j} v^{\Gamma_{F}-\Gamma_{j}}\left(\Delta_{1}\right)^{\mu_{1, j}}\left(\Delta_{2}\right)^{\mu_{2, j}} \ldots\left(\Delta_{k}\right)^{\mu_{k, j}} \equiv 0
$$

which can in general have non-constant entire solutions, which therefore do not violate the Pang-Zalcman Lemma and thus do not lead to the required contradictions. For instance, consider $F[f]=f^{3}+\left(f^{\prime}\right)^{2} f^{\prime \prime \prime}-f^{\prime}\left(f^{\prime \prime}\right)^{2}$. From this, we get that $\Gamma_{F}=8, L=5$ and $\alpha=\frac{2}{3}$, and so

$$
\Psi=v^{5}+\Delta_{3}-\left(\Delta_{2}\right)^{2}
$$

Thus when applying Theorem 4.2.2, we have

$$
\Phi=v^{3}\left(\frac{1}{v}\right)^{\prime \prime}-\left(v^{2}\left(\frac{1}{v}\right)^{\prime}\right)^{2} \equiv 0,
$$

which is solved by the non-constant entire solution family $a e^{b z}$ for non-zero constants $a, b$, and this family is not normal. It is also worth noting that these entire solutions are generally the exceptional case, and rely on specific values of coefficients. For instance, if we take $G[f]=f^{3}+\left(f^{\prime}\right)^{2} f^{\prime \prime \prime}+f^{\prime}\left(f^{\prime \prime}\right)^{2}$, this has the same structure as $F$ but different coefficients. The associated differential equation (5.3.4) has solutions of the form $v=$ $(a \sqrt{b+2 z})^{-1}$ for constants $a, b$, and so has no non-constant entire solutions. 


\section{Chapter 6}

\section{Integer points of meromorphic}

\section{functions}

In this chapter, we work from a half-plane result of Fletcher and Langley [12] and show that if $f$ is an integer-valued function on some subset of the natural numbers of positive lower density, and is meromorphic of sufficiently small exponential type in the plane, then $f$ is a polynomial. This work is due to be published in Proceedings of the Edinburgh Mathematical Society [8].

\subsection{Introduction and result}

An integer-valued function is one such that $f(\mathbb{Z}) \subseteq \mathbb{Z}$, a simple example being a polynomial with integer coefficients, or $\sin (\pi z)$. Research in this field generally focusses on functions which are integer-valued on some subset of $\mathbb{Z}$. Pólya proved an early result in this field. 


\section{Proposition 6.1.1 [26]}

Let $f$ be entire, taking integer values on $\mathbb{N} \cup\{0\}$, and suppose that

$$
\limsup _{r \rightarrow \infty} \frac{M(r, f)}{2^{r}}<1
$$

where $M(r, f)$ is the maximum modulus function of $f$. Then $f$ is a polynomial.

Langley in [20] later showed that the lim sup cannot be replaced by a lim inf. A corollary to Pólya's result is that $2^{z}$ is the slowest growing transcendental entire function to take integer values on the non-negative integers. Pólya further showed that

\section{Proposition 6.1.2 [26]}

Let $f$ be an entire function such that $f(n) \in \mathbb{Z}$ for $n=0,1,2, \ldots$ and

$$
\limsup _{r \rightarrow \infty} \frac{\log M(r, f)}{r} \leq \alpha \leq \log 2
$$

Then there exist polynomials $P_{j}(z)$ such that

$$
f(z)=P_{1}(z) 2^{z}+P_{2}(z)
$$

This was later improved to $\alpha \leq \log 2+\frac{1}{1500}$ by Selberg in [28], then further by Pisot in $[25]$.

Fletcher and Langley proved a half-plane analogue to Pólya's result [12],

\section{Proposition 6.1.3 [12]}

Let $d, J$ and $\lambda$ satisfy

$$
0<d<1, \quad J \in \mathbb{N}, \quad \lambda>0, \quad \frac{16}{J}\left(1+\log \left(1+\frac{J}{2}\right)\right)+8(J-1) \lambda<d^{2} .
$$

Let $E \subset \mathbb{N}$ have lower density

$$
\underline{D}(E)=\liminf _{n \rightarrow \infty} \frac{|E \cap\{1, \ldots, n\}|}{n}>d,
$$


let $f$ be analytic of exponential type less than $\lambda$ in the closed right half plane, and assume that $f(n) \in \mathbb{Z}$ for every $n \in E$. Then $f$ is a polynomial.

Further related work may be found in [9], [11], [22], [27] and [34], among others. However, there does not appear to have been any research into whether an analogue of Pólya's result can be obtained for meromorphic functions. In this chapter, we generalise Fletcher and Langley's result to meromorphic functions, following the general method of their proof, which was in turn based on a method of Waldschmidt [32]. Our result is restricted to functions which are meromorphic in the whole plane rather than a half plane, mainly due to the Poisson-Jensen formula being significantly easier to use in the whole plane.

\section{Theorem 6.1.4}

Given $d \in(0,1)$, there exists some $\lambda=\lambda(d)>0$ with the following property. Let $f$ be meromorphic in the plane, taking integer values on some set $E \subseteq \mathbb{N}$ of positive lower density $d_{0}>d$, with $T(r, f) \leq \lambda r$ for all $r \geq r_{0}$. Then $f$ is a polynomial.

We will calculate how small $\lambda$ needs to be in the appendix (6.4).

\subsection{Lemmas}

To begin with, we will prove some lemmas. The first is an elementary result comparing the integrated and unintegrated counting functions. 


\section{Lemma 6.2.1}

Let $0<s<S$, and let $h$ be a meromorphic function on the set $|z| \leq S$. Then

$$
N(S, h) \geq n(s, h) \log \frac{S}{s}+n(0, h) \log s
$$

This is a well-known result, and so we omit the proof. The next lemma is found in many texts, including [17], where it is presented as a mass distribution result. A more elementary proof can be found in [18].

\section{Lemma 6.2.2 - The (Boutroux-)Cartan Lemma}

Let $z_{1}, \ldots, z_{n} \in \mathbb{C}$, and $\gamma>0$. Then

$$
V(z)=\sum_{j=1}^{n} \log \left|z-z_{j}\right|>n \log \gamma
$$

for all $z$ outside a union $U$ of open discs of total radius at most $6 \gamma$.

Remark: We may assume that the discs are disjoint, since if some point $z_{0}$ is within two discs, of radius $r_{1}$ and $r_{2}$ respectively, we may choose a new disc of radius $r_{3}<r_{1}+r_{2}$ that encloses both original discs. We may also assume that each disc contains at least one $z_{j}$, as otherwise (6.2.1) applies on the boundary of that disc, and since $V$ is harmonic inside the disc, we may extend (6.2.1) to the interior. We may therefore assume that there are at most $n$ discs.

We now apply Boutroux-Cartan to give a bound on the logarithm of the modulus of a function in terms of its Nevanlinna characteristic. 


\section{Lemma 6.2.3}

Let $m \geq 0, s \geq 1,0<\varepsilon \leq 1$, and let $h$ be meromorphic on the set $|z| \leq 8 s$ with at least $m$ distinct zeros in $|z| \leq s$. Then

$$
\log |h(z)| \leq\left(6-\frac{\log \varepsilon}{\log 2}\right) T(8 s, h)+m \log \frac{6}{7}
$$

for all $|z| \leq 2 s$ lying outside a union $U$ of at most $n(4 s, h)$ open discs of total radius at most $24 \varepsilon s$.

Remark: A disc of radius $s>0$ contains at most $1+2 s$ distinct integers, and so the number of integers in $U$ is at most the number of discs plus double the total radius.

\section{Proof:}

Let $S=4 s$ and $n=n(4 s, h)$, and further let $b_{1}, \ldots, b_{n}$ be the poles of $h$ in $|z| \leq S$, repeated according to multiplicity. If $m>0$, let $a_{1}, \ldots, a_{m}$ be distinct zeros of $h$ in $|z| \leq s$. Finally, define the function $g$ by

$$
g(z)=h(z) \prod_{j=1}^{m} \frac{S^{2}-\overline{a_{j}} z}{S\left(z-a_{j}\right)} \prod_{k=1}^{n} \frac{z-b_{k}}{S}
$$

where an empty product is taken as 1 . Thus $g$ is analytic on $|z| \leq S$. Also, for $|z|=S$, we have

$$
\left|\frac{S^{2}-\overline{a_{j}} z}{S\left(z-a_{j}\right)}\right|=1 \quad \text { and } \quad\left|\frac{z-b_{k}}{S}\right| \leq 2
$$

and so,

$$
T(S, g)=m(S, g) \leq m(S, h)+n(S, h) \log 2 .
$$

Since $S>1$, we have by Lemma 6.2.1

$$
N(2 S, h) \geq n(S, h) \log 2,
$$


and so

$$
T(S, g) \leq m(S, h)+N(2 S, h) \leq 2 T(2 S, h)
$$

Thus, by the standard comparison between the maximum modulus and characteristic functions for functions analytic on a disc centred at the origin (1.2.15), we have for $|z| \leq 2 s=S / 2$

$$
\log |g(z)| \leq \frac{S+\frac{S}{2}}{S-\frac{S}{2}} T(S, g)=3 T(S, g) \leq 6 T(2 S, h)=6 T(8 s, h)
$$

Also in this region we have $\left|z-a_{j}\right| \leq 3 s$ and $\left|S^{2}-\overline{a_{j}} z\right| \geq 14 s^{2}$, and so

$$
\left|\frac{S\left(z-a_{j}\right)}{S^{2}-\overline{a_{j}} z}\right| \leq \frac{4 s 3 s}{14 s^{2}}=\frac{6}{7}
$$

We apply Boutroux-Cartan with $\gamma=\varepsilon S$ to find that outside a union $U$ of at most $n$ open discs of total radius at most $24 \varepsilon s$,

$$
\sum_{k=1}^{n} \log \left|z-b_{k}\right| \geq n \log 4 \varepsilon s
$$

Thus, for $|z| \leq 2 s, z \notin U$,

$$
\begin{aligned}
\log |h(z)| & =\log |g(z)|+\sum_{j=1}^{m} \log \left|\frac{S\left(z-a_{j}\right)}{S^{2}-\overline{a_{j}} z}\right|+\sum_{k=1}^{n} \log S-\sum_{k=1}^{n} \log \left|z-b_{k}\right| \\
& \leq 6 T(8 s, h)+m \log \frac{6}{7}-n \log 4 \varepsilon s+n \log 4 s \\
& =6 T(8 s, h)+m \log \frac{6}{7}-n \log \varepsilon
\end{aligned}
$$

where, by Lemma 6.2.1,

$$
n=n(4 s, h) \leq \frac{N(8 s, h)}{\log 2} \leq \frac{T(8 s, h)}{\log 2},
$$

from which the result follows. 
The following lemma allows us to say that if a function has some zeros in a certain segment of the real line, then it has more zeros in a larger segment. Repeated application of this allows us to cover the entire range $[1, \infty)$.

\section{Lemma 6.2.4}

Given $d \in(0,1)$, there exists $\vartheta=\vartheta(d)>0$ with the following property. Let $R \geq 1$, $E \subseteq \mathbb{N}$ be such that $|E \cap[1, r]| \geq d r$ for all $r \geq R$, let $F(E) \subseteq \mathbb{Z}$ where $F$ is meromorphic in $\mathbb{C}$ and has at least $d R / 2$ distinct zeros in $E \cap[1, R]$, and $T(r, F) \leq \vartheta r$ for all $r \geq R$. Then $F$ has at least $d R$ distinct zeros in $E \cap[1,2 R]$.

Proof:

Let $\varepsilon=d / 96$, and let $m$ be the least integer such that $m \geq d R / 2$. We apply Lemma 6.2.3 with $h=F$ and $s=R$ to give, for $|z| \leq 2 R$ outside some union $U$ of at most $n(4 R, F)$ open discs of total radius at most $d R / 4$,

$$
\log |F(z)| \leq\left(6-\frac{\log \varepsilon}{\log 2}\right) 8 \vartheta R+\frac{d R}{2} \log \frac{6}{7}
$$

It is easy to check that with small enough $\vartheta$, this gives $\log |F(z)|<0$. Further, by our earlier remark on Lemma 6.2.3, $U$ encloses at most

$$
n(4 R, F)+48 \varepsilon R \leq \frac{T(8 R, F)}{\log 2}+48 \varepsilon R \leq\left(\frac{8 \vartheta}{\log 2}+\frac{d}{2}\right) R
$$

integers. Given that $|E \cap[1,2 R]| \geq 2 d R$, it is clear that if $\vartheta$ is small enough then after removing any points of $E \cap[1,2 R] \cap U$ we are left with at least $d R$ integers in $(E \cap[1,2 R]) \backslash U$, which, since $F(E) \subseteq \mathbb{Z}$ and $|F(z)|<1$ at these points, must be zeros of $F$. 
We now proceed to several lemmas from [12], which form the main structure of the proof. We first create a sequence of polynomials, then look at an application of linear forms, and finally note that if a function is algebraic on a half plane and takes integer values, then it is a polynomial.

\section{Lemma 6.2.5 [12]}

Define polynomials $p_{0}, p_{1}, \ldots$ by

$$
p_{0}(z)=1, \quad p_{1}(z)=z, \quad p_{h}(z)=\frac{z(z-1) \ldots(z-h+1)}{h !}(h=2,3, \ldots) .
$$

Then for $R>0, H \in \mathbb{N}, 0 \leq h \leq H$ and $|z| \leq R$, we have $p_{h}(\mathbb{Z}) \subseteq \mathbb{Z}$ and

$$
\left|p_{h}(z)\right| \leq e^{H}\left(\frac{R}{H}+1\right)^{H} .
$$

Proof:

It is easy to see that $p_{h}(\mathbb{Z}) \subseteq \mathbb{Z}$. For the inequality, we write

$$
\left|p_{h}(z)\right| \leq \frac{(R+H)^{h}}{h !} \leq \frac{H^{h}}{h !}\left(\frac{R}{H}+1\right)^{H} \leq e^{H}\left(\frac{R}{H}+1\right)^{H}
$$

Lemma 6.2.6 $[12]$

Let $B \geq 1$ and $N \geq 2$ be integers. Suppose that $L_{1}, \ldots, L_{m}$ are linear forms in the $n$ variables $x_{1}, \ldots, x_{n}$, with real coefficients $a_{j, k}$ for $j=1, \ldots, m$ and $k=1, \ldots, n$, such that $L_{j}=a_{j, 1} x_{1}+\ldots+a_{j, n} x_{n}$. Suppose further that $n>m$ and

$$
\max _{j, k}\left|a_{j, k}\right| \leq B
$$

Then there exist integers $x_{1}, \ldots, x_{n}$, not all zero, such that for $j=1, \ldots, m$ and $k=$ $1, \ldots, n$

$$
\left|L_{j}\right| \leq \frac{1}{N} \quad \text { and } \quad\left|x_{k}\right| \leq 2(2 n B N)^{\frac{m}{n-m}}
$$


Lemma 6.2.7 $[12]$

Let the algebraic function $f$ be analytic on the half plane $\operatorname{Re}(z) \geq 0$, and satisfy $f(E) \subseteq \mathbb{Z}$ for some set $E \subseteq \mathbb{N}$ of positive lower density. Then $f$ is a polynomial.

\subsection{Proof of Theorem 6.1.4}

Fix a large positive integer $J$, and given $J$ let $R$ be a large positive integer. How large $J$ must be will be determined later.

Apply Lemma 6.2 .3 with $h=f, m=0, s=R / 2$ and $\varepsilon=d / 96$ to give that, for $|z| \leq R$ outside a union $U$ of open discs of total radius at most $d R / 8$,

$$
\log |f(z)| \leq\left(6-\frac{\log \frac{d}{96}}{\log 2}\right) 4 \lambda R=\Lambda R .
$$

By (6.2.4), replacing $R$ with $R / 2$,

$$
|\mathbb{Z} \cap U| \leq \frac{T(4 R, f)}{\log 2}+24 \varepsilon R \leq\left(\frac{4 \lambda}{\log 2}+\frac{d}{4}\right) R<\frac{d R}{3}
$$

for small enough $\lambda$. Since $R$ is large we therefore have $m \geq d R / 2$ distinct integers $\alpha_{1}, \ldots, \alpha_{m} \in E \cap[1, R]$, where $m / J \in \mathbb{N}$, for which $f\left(\alpha_{j}\right) \in \mathbb{Z}$ and (6.3.1) is satisfied.

Now, set $n=2 m, H=n / J \in \mathbb{N}$, and form $n=H J$ functions

$$
g_{k}(z)=p_{\mu(k)}(z) f(z)^{\nu(k)}
$$

for $\mu=0,1, \ldots, H-1, \nu=0,1, \ldots, J-1$, where the $p_{\mu}$ are as in Lemma 6.2.5. Note that $H$ is dependent on $R$, but that $J$ is fixed. Let $a_{j, k}=g_{k}\left(\alpha_{j}\right) \in \mathbb{Z}$. We obtain the following estimate by Lemma 6.2.5 and (6.3.1):

$$
\begin{aligned}
\left|a_{j, k}\right| & =\left|g_{k}\left(\alpha_{j}\right)\right|=\left|p_{\mu(k)}\left(\alpha_{j}\right)\right|\left|f\left(\alpha_{j}\right)\right|^{\nu(k)} \\
& \leq e^{H}\left(\frac{R}{H}+1\right)^{H}\left(e^{\Lambda R}\right)^{J-1} \\
& =A(R) \leq\lceil A(R)\rceil=B(R) \leq 2 A(R),
\end{aligned}
$$


where $\lceil x\rceil$ is the smallest integer not less than $x$. We apply Lemma 6.2 .6 with $N=2$ and $n=2 m$ to give integers $A_{1}, \ldots, A_{n}$, not all zero, such that

$$
\sum_{k=1}^{n} A_{k} g_{k}\left(\alpha_{j}\right)=0
$$

for $j=1, \ldots, m$, and

$$
\left|A_{k}\right| \leq 8 n B \text {, where } B=B(R) .
$$

Now set

$$
F(z)=\sum_{k=1}^{n} A_{k} g_{k}(z) .
$$

$F$ is meromorphic, takes integer values on $E$ and is 0 at the $\alpha_{j}$ for $j=1, \ldots, m$. We now estimate $T(r, F)$ for each $r \geq R$. Note first that since the $p_{\mu}(z)$ are polynomials, all poles of $F$ must come from poles of $f$, and so

$$
N(r, F) \leq(J-1) N(r, f)
$$

Also, for non-negative $x_{1}, \ldots, x_{n}$,

$$
\log ^{+}\left(\sum_{k=1}^{n} x_{k}\right) \leq \log n+\max _{1 \leq k \leq n} \log ^{+}\left|x_{k}\right| .
$$

For $r \geq R$, we have by Lemma 6.2.5 that

$$
\begin{aligned}
\log |F(z)| & \leq \log n+\max _{1 \leq k \leq n,|z|=r}\left(\log ^{+}\left|A_{k} g_{k}(z)\right|\right) \\
& \leq \log n+\log 8 n B+H\left(1+\log \left(\frac{r}{H}+1\right)\right)+(J-1) \log ^{+}|f(z)|
\end{aligned}
$$

Thus, by integrating we obtain

$$
m(r, F) \leq \log n+\log 8 n B+H\left(1+\log \left(\frac{r}{H}+1\right)\right)+(J-1) m(r, f),
$$

and so

$$
\begin{aligned}
T(r, F) & \leq \log n+\log 8 n B+H\left(1+\log \left(\frac{r}{H}+1\right)\right)+(J-1) T(r, f) \\
& \leq \log n+\log 16 n A+H\left(1+\log \left(\frac{r}{H}+1\right)\right)+(J-1) T(r, f) .
\end{aligned}
$$


Now, for $r \geq R$, since $\Lambda>\lambda$ by (6.3.1) and $n=2 m \leq 2 r$ and $R$ is large, we have

$$
\begin{aligned}
T(r, F) \leq & 4 \log 2+2 \log n+\log \left(e^{H}\left(\frac{R}{H}+1\right)^{H} e^{(J-1) \Lambda R}\right)+ \\
& +H\left(1+\log \left(\frac{r}{H}+1\right)\right)+(J-1) T(r, f) \\
\leq & 4 \log 2+2 \log 2 r+H\left(1+\log \left(\frac{R}{H}+1\right)\right)+(J-1) \Lambda R+ \\
& +H\left(1+\log \left(\frac{r}{H}+1\right)\right)+(J-1) \lambda r \\
\leq & 2 H\left(1+\log \left(\frac{r}{H}+1\right)\right)+2(J-1) \Lambda r .
\end{aligned}
$$

By differentiation, it may be verified that $x^{-1}(1+\log (x+1))$ is decreasing for $x>0$. So, for $n=2 m \leq 2 R \leq 2 r$, this gives

$$
\frac{r}{H} \geq \frac{R}{H}=\frac{R J}{n}=\frac{R J}{2 m} \geq \frac{J}{2}
$$

and

$$
\begin{aligned}
2 H\left(1+\log \left(\frac{r}{H}+1\right)\right) & =2 r \frac{H}{r}\left(1+\log \left(\frac{r}{H}+1\right)\right) \\
& \leq 2 r \frac{2}{J}\left(1+\log \left(\frac{J}{2}+1\right)\right) \\
& =\frac{4 r}{J}\left(1+\log \left(\frac{J}{2}+1\right)\right) .
\end{aligned}
$$

Thus,

$$
T(r, F) \leq \frac{4 r}{J}\left(1+\log \left(\frac{J}{2}+1\right)\right)+2(J-1) \Lambda r
$$

and so we can say that for large enough $R$,

$$
T(r, F)<\vartheta r
$$

for $r \geq R$, where $\vartheta>0$ can be arbitrarily small provided that $\Lambda$ is small enough and $J$ large enough. We also have $F\left(\alpha_{j}\right)=0$ for $j=1, \ldots, m$ where $m \geq d R / 2$. We apply Lemma 6.2.4 to give at least $d R$ zeros of $F$ in $E \cap[1,2 R]$. We apply this repeatedly to give an infinite sequence of zeros of $F$ on the real line. Assume that $F(z) \not \equiv 0$. We have 
that $n\left(2^{t} R, 1 / F\right) \geq 2^{t-1} d R$, and so $n(r, 1 / F) \geq d r / 4$ for all $r \geq R$. By application of Lemma 6.2.1 we find $N(e r, 1 / F) \geq d r / 4$, thus $T(r, 1 / F) \geq d r / 4 e$, and so by the First Fundamental Theorem (1.3.1),

$$
T(r, F) \geq d r / 4 e-O(1)
$$

However, if $\vartheta$ is small enough, this is incompatible with (6.3.6). Hence, $F(z) \equiv 0$.

Now, recall from (6.3.3) and (6.3.4) that

$$
F(z)=\sum_{\nu=0}^{J-1}\left(\sum_{\mu=0}^{H-1} A_{\mu, \nu} p_{\mu}(z)\right) f(z)^{\nu},
$$

where at least one $A_{\mu, \nu}$ is non-zero, and where $p_{\mu}(z)$ has degree $\mu$. Thus, these polynomials cannot cancel each other out, hence $f$ is algebraic, and so must have only finitely many poles. Therefore there is some $x \in \mathbb{N}$ such that there are no poles in the half plane $\operatorname{Re}(z) \geq x$, so $f$ is analytic in this region. We apply Lemma 6.2 .7 to $f(z-x)$, giving that $f(z-x)$ is a polynomial here. From this, we conclude that $f(z)$ is polynomial in the half-plane $\operatorname{Re}(z) \geq x$, and thus by the identity theorem $f(z)$ must be a polynomial on the whole plane.

QED

\subsection{Appendix - How small is $\lambda(d)$ ?}

An obvious question to ask about this theorem is "how small must $\lambda$ be?" We will now calculate this. We make no claim as to how sharp these values are, but have sought to present a positive result in a reasonably accessible fashion.

We begin by calculating $\vartheta$ in Lemma 6.2.4. We use (6.2.3), substituting in $d / 96$ for $\varepsilon$, and noting that since we want $|F(z)|<1$ in order to force $F(\alpha)=0$ for $\alpha \in E \backslash U$, 
we require $\log |F(z)|<0$. Hence,

$$
\vartheta<\frac{d \log \frac{7}{6}}{16\left(6-\frac{\log (d / 96)}{\log 2}\right)}=\gamma(d) .
$$

We also require that $U$ encloses at most $d R$ integers, and so by (6.2.4) we need

$$
\left(\frac{8 \vartheta}{\log 2}+\frac{d}{2}\right) R \leq d R,
$$

which simplifies to

$$
\vartheta \leq \frac{d \log 2}{16} .
$$

We further require by (6.3.6) and (6.3.7) that

$$
\vartheta<\frac{d}{4 e} .
$$

However,

$$
\frac{d \log \frac{7}{6}}{16\left(6-\frac{\log (d / 96)}{\log 2}\right)}<\frac{d \log \frac{7}{6}}{96}<\frac{d}{48}<\frac{d \log 2}{16}<\frac{d}{4 e},
$$

hence both (6.4.2) and (6.4.3) are much looser bounds than (6.4.1) and so may be ignored.

We now move on to $\Lambda$. The proof of the theorem by (6.3.2) requires

$$
\lambda<\frac{d \log 2}{48} .
$$

It also requires by (6.3.1) and (6.3.5) that

$$
\vartheta=\frac{4}{J}\left(1+\log \left(\frac{J}{2}+1\right)\right)+2(J-1)\left(6-\frac{\log \frac{d}{96}}{\log 2}\right) 4 \lambda .
$$

Suppose we choose $J$ so large that

$$
\frac{4}{J}\left(1+\log \left(\frac{J}{2}+1\right)\right)<\frac{\gamma(d)}{2},
$$


and, given this $J$, choose $\lambda$ such that

$$
2(J-1)\left(6-\frac{\log \frac{d}{96}}{\log 2}\right) 4 \lambda<\frac{\gamma(d)}{2} .
$$

Then the pair $(J, \lambda)$ will satisfy (6.4.1). Further, we have

$$
\lambda<\frac{\gamma(d)}{96}<\frac{d \log \frac{7}{6}}{96^{2}}<\frac{d \log 2}{48}
$$

and so (6.4.4) holds. Solving these inequalities using Mathematica for $J$ in terms of $d$ produces the following new inequality:

$$
J>\frac{128 \log \frac{d}{6144}}{d \log \frac{7}{6} \log 2} W\left(\frac{d \log \frac{7}{6} \log 2 \exp \left(\frac{d \log \frac{7}{6} \log 2}{64 \log \frac{d}{6144}}-1\right)}{64 \log \frac{d}{6144}}\right)-2
$$

where $W$ is the Lambert $\mathrm{W}$-function. Again using Mathematica, solving for specific values of $d$ gives the following results for $J$ and $\lambda$ :

$$
\begin{array}{lll}
d=1 & J \gtrsim 130,000 & \lambda \lesssim 2.9 \times 10^{-11} \\
d=0.5 & J \gtrsim 290,000 & \lambda \lesssim 5.6 \times 10^{-12} \\
d=0.1 & J \gtrsim 2,000,000 & \lambda \lesssim 1.2 \times 10^{-13} \\
d=0.01 & J \gtrsim 28,000,000 & \lambda \lesssim 5.8 \times 10^{-16}
\end{array}
$$

Note that $d=1$ is essentially meaningless here, as we require our set $E$ to have lower density greater than $d$, but it provides a useful upper bound.

By comparison, using a similar process on the Fletcher-Langley result (Proposition 6.1.3) yields a maximal value of $\lambda$ of roughly $3.6 \times 10^{-4}$ for $d$ close to 1 .

\subsection{Appendix - A thought on further work}

We conclude by asking a question about a topic which does not appear to have been the subject of any research: can any results be obtained by restricting what integer values 
may be taken? Specifically, for $n \in\{1,2,4\}$, is $2^{n z}$ the slowest-growing transcendental meromorphic function taking only $n^{\text {th }}$ powers of integers on the natural numbers? Pólya's result (the corollary to Proposition 6.1.1) proves this for $n=1$, but beyond this the way forward is unclear. The restriction to only three integer values of $n$ is due to the sine function: for odd $n \geq 3, \sin (\pi z / 2)$ has the required properties and is smaller than $2^{3 z}$, and for even $n \geq 6, \sin (\pi z)$ is sufficient. 


\section{Bibliography}

[1] W. Bergweiler, On the zeros of certain homogeneous differential polynomials, (Archiv der Mathematik, 64, no. 3, 1995, pp199-202).

[2] W. Bergweiler, Bloch's principle (Computational Methods and Function Theory, 6, no. 1,2006, pp77-108).

[3] W. Bergweiler and J. K. Langley, Nonvanishing derivatives and normal families (Journal d'Analyse Mathématique, 91, 2003, pp353-367).

[4] W. Bergweiler and J. K. Langley, Multiplicities in Hayman's Alternative (Journal of the Australian Mathematical Society, 78, 2005, pp37-57).

[5] M. Buck, Pairs of non-homogeneous linear differential polynomials (Computational Methods and Function Theory, 11, no. 1, 2011, pp283-300).

[6] M. Buck, Non-linear homogeneous differential polynomials, (Computational Methods and Function Theory, 12, no. 1, 2012, pp145-150).

[7] M. Buck, A theorem on homogeneous differential polynomials, (Results in Mathematics, 63, no. 3, 2013, pp805-815).

[8] M. Buck, Integer points of meromorphic functions, (Proceedings of the Edinburgh Mathematical Society, to appear). 
[9] R. C. Buck, Integral valued entire functions, (Duke Mathematical Journal, 15, no.4, 1948, pp879-891).

[10] J. Clunie, On integral and meromorphic functions, (Journal of the London Mathematical Society, 37, 1962, pp17-27).

[11] A. N. Fletcher, Gaussian integer points of functions analytic in a half plane, (Mathematical Proceedings of the Cambridge Philosophical Society, 145, no.2, 2008, pp257$272)$.

[12] A. N. Fletcher and J. K. Langley, Integer points of analytic functions in a half plane, (Proceedings of the Edinburgh Mathematical Society (2), 52, no.3, 2009, pp619-630).

[13] G. Frank, Über die Nullstellen von linearen Differentialpolynomen mit meromorphen Koeffizienten, in Complex methods on partial differential equations, (Mathematics Research Series, volume 53, 1989, pp39-48).

[14] G. Frank, W. Hennekemper and G. Polloczek, Über die Nullstellen meromorpher Funktionen and ihrer Ableitungen, (Mathematische Annalen, 225, 1977, pp145-154).

[15] G. Frank and J. K. Langley, Pairs of linear differential polynomials, (Analysis, 19, 1999, pp173-194).

[16] W. K. Hayman, Meromorphic Functions, (Oxford, Clarendon Press, 1964).

[17] W. K. Hayman, Subharmonic Functions Volume 2, (London, Academic Press, 1989).

[18] I. Laine, Complex Analysis 2, (http://www.joensuu.fi/matematiikka/kurssit/complex/, retrieved August 2011). 
[19] J. K. Langley, Proof of a conjecture of Hayman concerning $f$ and $f^{\prime \prime}$, (Journal of the London Mathematical Society, (2) 48, 1993, pp500-514).

[20] J. K. Langley, Integer points of entire functions, (Bulletin of the London Mathematical Society, 38, 2006, pp239-249).

[21] J. K. Langley, Pairs of non-homogeneous linear differential polynomials, (Proccedings of the Royal Society of Edinburgh, 136A, 2006, pp785-794).

[22] J. K. Langley, Integer-valued analytic functions in a half-plane, (Computational Methods and Function Theory, 7, no.2, 2007, pp433-442).

[23] J. K. Langley, An inequality of Frank, Steinmetz and Weissenborn, (Kodai Mathematical Journal, 34, 2011, pp383-389).

[24] E. Mues and N. Steinmetz, The theorem of Tumura-Clunie for meromorphic functions, (Journal of the London Mathematical Society (2), 23, 1981, pp113-122).

[25] C. Pisot, Über ganzwertige ganze Funktionen (Jahresbericht der Deutsche Mathematiker-Vereinigung, 52, 1942, pp95-102).

[26] G. Pólya, Über ganze ganzwertige Funktionen, (Nachrichten von der Gesellschaft der Wissenschaften zu Göttingen, c1920, pp1-10).

[27] R. M. Robinson, Integer-valued entire functions, (Transactions of the American Mathematical Society, 153, 1971, pp451-468).

[28] A. Selberg, Über ganzwertige ganze transzendente Funktionen, (Archiv for Mathematik og Naturvidenskab, 44, 1941, pp45-52).

[29] A. P. Singh, On order of homogeneous differential polynomials (Indian Journal of Pure and Applied Mathematics, 16, no. 7, 1986, pp791-795). 
[30] N. Steinmetz, On the zeros of a certain Wronskian, (Bulletin of the London Mathematical Society, 20, 1988, pp525-531).

[31] K. Tohge, On the zeros of a homogeneous differential polynomial of a meromorphic function, (Kodai Mathematical Journal, 16, no. 3, 1993, pp398-415).

[32] M. Waldschmidt, Integer valued entire functions on Cartesian products, (Number Theory in Progress, 1, 1999, pp553-576).

[33] A. Whitehead, Differential Equations and Differential Polynomials in the Complex Plane, (PhD thesis, University of Nottingham, 2002).

[34] K. Yoshino, Polya's theorem for nonentire functions, (Rendiconti del Circolo Matematico di Palermo (2), no.3, 1984, pp385-395).

[35] L. Zalcman, Normal families: new perspectives, (Bulletin (New Series) of the American Mathematical Society, 35, 1998, pp215-230).

[36] Z. L. Zhang and W. Li, Tumura-Clunie's theorem for differential polynomials, (Complex Variables. Theory and Application. An International Journal, 25, 1994, pp97$105)$. 